\title{
Relationship between speech-evoked neural responses and perception of speech in noise in older adults
}

Running title: Neural responses to speech-in-noise in elderly

Authors: Guangting Mai ${ }^{1}$, Jyrki Tuomainen ${ }^{2}$, Peter Howell ${ }^{1}$

${ }^{1}$ Department of Experimental Psychology, Division of Psychology and Language Sciences, University College London, London, WC1H 0AP

${ }^{2}$ Department of Speech, Hearing and Phonetic Sciences, Division of Psychology and Language Sciences, University College London, London, WC1N 1PF

Corresponding author: Peter Howell, Department of Experimental Psychology, Division of Psychology and Language Sciences, University College London, London, WC1H 0AP (p.howell@ucl.ac.uk)

Key words: speech-in-noise; older adults; phase-locking values; frequency-following responses 


\section{ABSTRACT}

Speech-in-noise (SPIN) perception involves neural encoding of temporal acoustic cues. Cues include temporal fine structure (TFS) and envelopes that modulate at syllable (Slow-rate ENV) and fundamental frequency $\left(\mathrm{F}_{0}\right.$-rate $\left.\mathrm{ENV}\right)$ rates. Here the relationship between speech-evoked neural responses to these cues and SPIN perception was investigated in older adults. Theta-band phase-locking values (PLV) that reflect cortical sensitivity to Slow-rate ENV and peripheral/brainstem frequency-following responses phase-locked to $\mathrm{F}_{0}$-rate $\mathrm{ENV}$

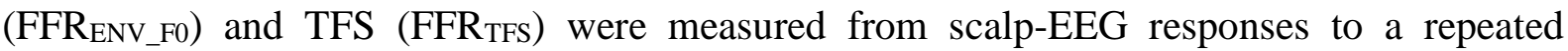
speech syllable in steady-state speech-shaped $(\mathrm{SpN})$ and 16-speaker babble $(\mathrm{BbN})$ noises. The results showed that: 1) SPIN performance and PLV were significantly higher under SpN than $\mathrm{BbN}$, implying differential cortical encoding may serve as the neural mechanism of SPIN performance that varies as a function of noise types; 2) PLV and FFR $_{\mathrm{TFS}}$ at resolved harmonics were significantly related to good SPIN performance, supporting the importance of phase-locked neural encoding of Slow-rate ENV and TFS of resolved harmonics during SPIN

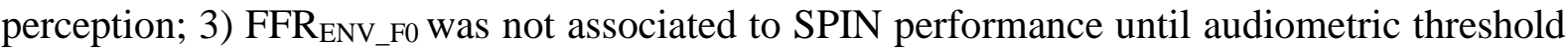
was controlled for, indicating that hearing loss should be carefully controlled when studying the role of neural encoding of $\mathrm{F}_{0}$-rate $\mathrm{ENV}$. Implications are drawn with respect to fitting auditory prostheses. 


\section{INTRODUCTION}

Perception of speech-in-noise (SPIN) requires the target speaker's voice to be separated from background noises. This can be achieved by processing temporal acoustic cues (Moore, 2008). There are three temporal components of speech signals that provide different information: 1) slowly-varying envelope (Slow-rate ENV) that modulates at rates of $<10 \mathrm{~Hz}$ and represents syllable structure (Rosen, 1992; Greenberg et al., 2003). Slow-rate ENV plays an essential role in speech perception both in quiet (Shannon et al., 1995; Arai et al., 1999) and in noise (Drullman et al., 1994); 2) The envelope that modulates at rates around $\mathrm{F}_{0}$ ( $\mathrm{F}_{0}$-rate ENV) and provides information about voicing and pitch (Rosen, 1992). Tracking the pitch of the target speaker is critical for segregating the target voice from background sounds (Bregman, 1994; Bird and Darwin, 1998; Binns and Culling, 2005); 3) Temporal fine structure (TFS) that fluctuates at rates above $\mathrm{F}_{0}$ (Rosen, 1992; Moore, 2008). TFS is important for perception of formant structure (Moore, 2014) as well as voicing and pitch (Rosen, 1992; Smith et al., 2002). SPIN perception is significantly better when TFS is present than when it is absent (Zeng et al., 2005; Stickney et al., 2007; Eaves et al., 2011). Neurophysiological tracking of these cues is also related to speech perception. For example, the degree of neural phase-locking to Slow-rate ENV is associated with speech intelligibility (Ahissar et al., 2001; Peelle et al., 2012; Doelling et al., 2014). Also, magnitudes of peripheral/subcortical speech-evoked frequency-following responses (FFRs) that are phase-locked to $\mathrm{F}_{0}$-rate ENV (FFR $_{\text {ENV_Fo) (Song et al., 2010) and TFS (FFR }}$ TFS) (Fujihira and Shiraishi, 2015) correlate with SPIN performance.

Older adults experience more difficulties in SPIN perception than normal-hearing 
younger adults (Moore, 2014). Behavioral studies suggest that this may be attributable to age-related deterioration in processing of TFS (Lorenzi et al., 2006; Hopkins, Moore and Stone, 2008; Hopkins and Moore, 2011). Neurophysiological studies show that FFR ENV_F0 and FFR $_{\text {TFS }}$ have smaller magnitudes in older, than in younger, adults (Anderson et al., 2012; Presacco et al., 2016). This suggests that poorer neuro-temporal encoding of $F_{0}$-rate ENV and TFS by older adults may account for impaired SPIN performance. Despite these findings, the relative importance of neural processing of $\mathrm{F}_{0}$-rate ENV and TFS remains obscure. While Anderson et al. (2011) showed that FFR_ENV_F0 was associated with SPIN ability in older adults, this result did not replicate (Schoof and Rosen, 2016). Recent studies report that in

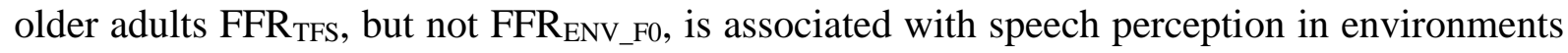
where there is reverberation (Fujihira and Shiraishi, 2015). This highlights the importance of neural encoding of TFS, compared to that of $\mathrm{F}_{0}$-rate ENV, during SPIN perception (Marsh and Campbell, 2016). Furthermore, since these studies (Anderson et al., 2011, 2012; Fujihira and Shiraishi, 2015; Presacco et al., 2016; Schoof and Rosen, 2016) were conducted in older adults with relatively normal hearing (hearing thresholds $\leq 30 \mathrm{~dB} H \mathrm{HL}$ at frequencies $\leq 4 \mathrm{kHz}$ ), they do not reflect the significant variability in audiometric hearing in aging populations (Gopinath et al., 2009; Humes et al., 2010).

The present hypothesis is that SPIN performance is associated more with phase-locked neural encoding of Slow-rate ENV and TFS compared to that of $\mathrm{F}_{0}$-rate ENV in older adults who vary in audiometric profiles. This is based on the observations that Slow-rate ENV is critical for speech perception both in quiet and in noise (Drullman et al., 1994; Shannon et al., 1995; Arai et al., 1999) and impaired sensitivity to TFS, rather than $\mathrm{F}_{0}$-rate ENV, significantly 
degrades SPIN performance (Stickney et al., 2007). To test the hypothesis, behavioral and neural assessments were conducted on healthy older adult participants in a sample with significant inter-individual variability in their audiometric profiles. Participants completed a SPIN task in which speech reception thresholds were measured under two types of noise: steady-state speech-shaped noise $(\mathrm{SpN})$ and 16-speaker babble noise $(\mathrm{BbN})$, where background speakers in $\mathrm{BbN}$ had similar voices characteristics to the target voice. SpN serves as a steady-state energetic masker, whilst $\mathrm{BbN}$ constitutes an envelope-modulated energetic masker as well as an informational masker that leads to phonetic interference. Rosen et al. (2013) showed that speech perception is better under $\mathrm{SpN}$ than $\mathrm{BbN}$ and that $\mathrm{BbN}$ is a particularly challenging form of noise for older adults (Helfer and Freyman, 2008; Schoof and Rosen, 2014). Participants listened to a repeated syllable in quiet, $\mathrm{SpN}$ and $\mathrm{BbN}$, whilst neural activity was recorded concurrently over scalp-EEG electrodes. Speech-evoked FFRs, which reflect peripheral/brainstem phase-locked encoding of $\mathrm{F}_{0}$-rate ENV and TFS (Aiken and Picton, 2008), were obtained. Cortical activity was represented by theta-band phase-locking values (PLVs) that reflect neural sensitivities to Slow-rate ENV (Luo and Poeppel, 2007; Howard and Poeppel, 2010). Signal-to-noise ratios (SNRs) of the acoustic stimuli in $\mathrm{SpN} / \mathrm{BbN}$ during the neural recording were set at either 7 or $-1 \mathrm{~dB}$, with the former $(7 \mathrm{~dB})$ being the SNR at which speech is reasonably recognizable while the latter $(-1 \mathrm{~dB})$ is the SNR that is relatively more challenging for SPIN perception (see Methods). The relationship between the neural measures and SPIN performance was then examined. It was predicted that the neural measures, especially those recorded under the challenging SNR, would correlate with the behavioral differences across noise types and individuals, and that the 
neural-behavioral relationship would further elucidate the underlying neural mechanisms of SPIN perception in older adults.

\section{METHODS}

\section{A. Participants}

Participants comprised 47 older healthy adults (aged 53 to 76 years, 17 males and 30 females). All were native English speakers who had lived in Britain for at least 25 years. No clinically-diagnosed neurological diseases, language-related or psychiatric problems were reported. All participants identified $100 \%$ of the words in the speech perception task correctly when SNR was $8 \mathrm{~dB}$. Twelve participants used hearing aids (HAs, either monaural or binaural). They wore their HAs with their usual settings and these were kept the same throughout the experiment. Fig. 1 shows the pure-tone audiograms (PTA) for frequencies between 0.25 and $8 \mathrm{kHz}$ of the non-HA participants measured via a MAICO MA41 Audiometer (MAICO Diagnostics, Germany). Inter-individual variability in hearing thresholds occurred particularly at high frequencies $(\geq 3 \mathrm{kHz})$, consistent with the distributions of audiometric profiles in aging populations (Gopinath et al., 2009; Humes et al., 2010). Fig. 1C shows the free-field PTAs of the HA users. They were measured by presenting pure tones via a Fostex 6301B loudspeaker (Canford Group Ltd, UK) at 0 degree azimuth positioned 1 meter away from the participants' ears. The sounds were controlled by the experimenter using a ThinkPad T430i laptop (Lenovo Group Ltd). Sound pressure levels (SPL) at 1 meter distance were measured and were then converted to hearing level (dB HL) for each frequency according to the ISO calibration guideline for free-field PTAs (ISO 
389-7-2005, British Society of Audiology, 2008). The free-field PTA confirmed that the HAs were functioning. However PTA does not precisely predict audibility of input stimuli with the current procedure because non-linear intensity gains are common in contemporary HAs (Levitt, 2007). Hence, free-field PTAs were not used in subsequent analyses.

A

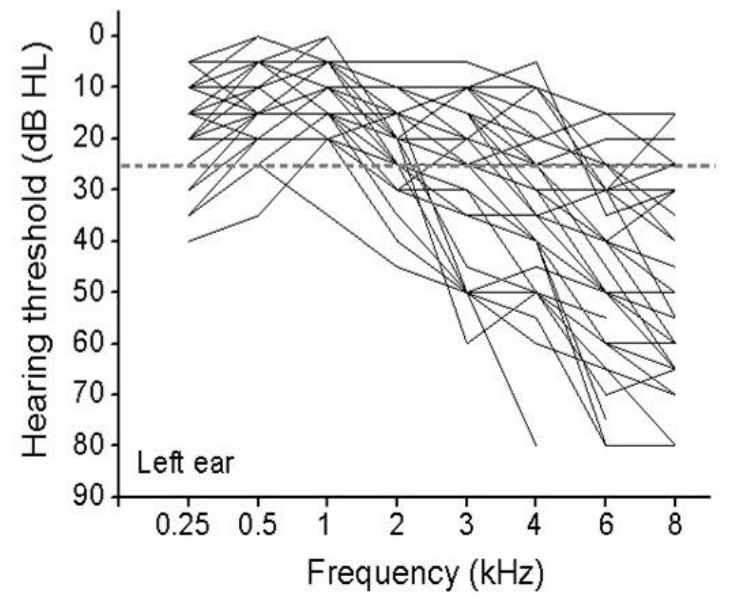

B

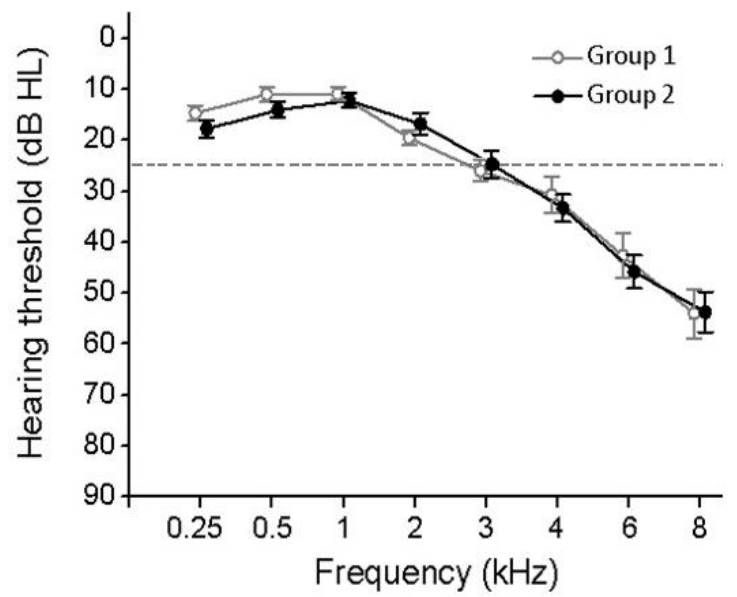

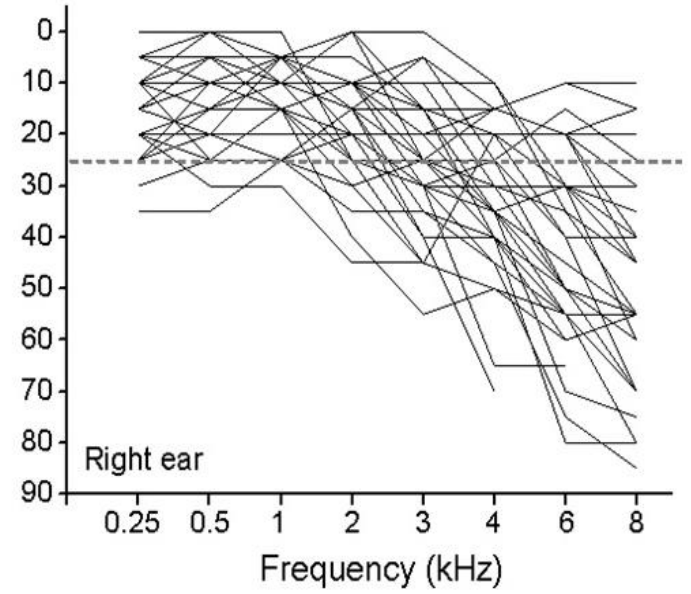

C

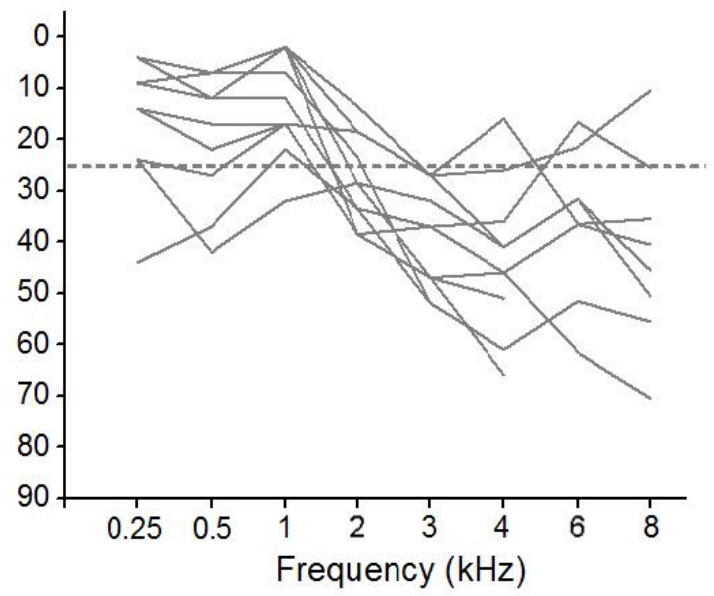

Figure 1. Pure-tone audiograms (PTAs) of the 35 non-HA participants. (A) Individual PTAs (left: left ear; right: right ear) of all non-HA participants. There was one participant's data at 6 $\mathrm{kHz}$ (on both ears) and six participants' data at $8 \mathrm{kHz}$ (two at both ears and four at either left 
or right ear) that were not plotted, because their hearing thresholds were $>85 \mathrm{~dB}$ HL and not measurable at these frequencies. (B) PTAs (collapsing the left and right ears) averaged across all non-HA participants (open and filled circles are data from for Group 1 and Group 2, respectively). When points were not measurable, data values were set at the highest possible value $(85 \mathrm{~dB})$ when average values and standard errors (SEs) were calculated. Error bars denote the SEs. (C) Free-field PTAs for HA users. There were three participants' data at 6 and $8 \mathrm{kHz}$ that were not plotted, because their hearing thresholds were $>85 \mathrm{~dB}$. The dashed lines indicate the normal hearing threshold level (25 dB HL).

Participants were randomly assigned to one of two groups, with the proviso that each group had six HA participants. The syllable stimuli were presented at different SNRs to the two participant groups when neural measurements were made (see TABLE I). There were 23 participants (59 to 76 years old; mean $\pm \mathrm{SE}=68.22 \pm 1.01 ; 8$ males) in Group 1 and 24 participants (53 to 75 years old; mean $\pm \mathrm{SE}=67.33 \pm 1.03$; 9 males) in Group 2. There were no significant differences in ages $(p=0.542)$ nor hearing thresholds in non-HA participants (averaged across 0.25 to $4 \mathrm{kHz}$ and across both ears) ( $p=0.693$ ) between the two groups. The neural data and neural-behavioral relationship of the two groups were analyzed separately.

TABLE I. Background noise types and SNR levels for Group 1 and 2 participants during the EEG recordings. During EEG recording, the stimuli in quiet background (NoN) were presented at the same level to both groups (all 47 participants), but the SNRs for noisy backgrounds $(\mathrm{SpN}$ and $\mathrm{BbN})$ differed in level across Groups 1 and $2(7 \mathrm{~dB}$ and $-1 \mathrm{~dB}$ 
respectively).

\begin{tabular}{cccc}
\hline SNR & $+\infty \mathrm{dB}$ & $7 \mathrm{~dB}$ & $-1 \mathrm{~dB}$ \\
& $(\mathrm{NoN})$ & $(\mathrm{SpN}$ and BbN $)$ & $(\mathrm{SpN}$ and BbN $)$ \\
\hline & & Group 1 & Group 2 \\
Participants & All 47 participants & (23 participants $)$ & (24 participants $)$ \\
\hline
\end{tabular}

\section{B. Speech-in-noise (SPIN) perception task}

Speech Reception Thresholds (SRTs) (SNR level for 50\% word correct, as in Plomp and Mimpen, 1979) were measured when participants recognized words within sentences under two different types of background noise, steady-state speech-shaped noise ( $\mathrm{SpN}$ ) and 16-speaker babble noise $(\mathrm{BbN})$. Target sentences were pre-recorded BKB sentences (Bench et al., 1979) spoken by a male British English speaker whose $\mathrm{F}_{0}$ ranged between 80 and $200 \mathrm{~Hz}$. Each sentence was meaningful and included three key words (content words), e.g., for 'The clown has a funny face', the key words were 'clown', 'funny' and 'face'. The BKB sentences were used rather than more complex ones such as IEEE sentences (Rothauser et al., 1969) in order to minimize effects of individual variability in working memory on speech perception performance. The $\mathrm{BbN}$ was a mixture of 16 different utterances spoken by 16 male British English speakers who had similar $\mathrm{F}_{0} \mathrm{~S}$ and voice quality to the target speaker. The $\mathrm{SpN}$ was created by randomizing the phases in the long-term spectrum of $\mathrm{BbN}$ before the spectrum was transformed back to the time domain. Consequently $\mathrm{SpN}$ had the same long-term spectral power distribution as $\mathrm{BbN}$ and relatively stable temporal properties (Rosen et al., 2013). The 
background noise always started about $500 \mathrm{~ms}$ prior to the onset of the target sentence and continued until the sentence ended.

Participants were seated in a sound-treated booth facing a Fostex 6301B loudspeaker (Canford Group Ltd, UK) at 0 degree azimuth positioned one-meter away from the their ears. The sentences were all presented at an intensity of $70 \mathrm{~dB}$ SPL at the 1 meter distance. Participants listened to different sets of 30 sentences in $\mathrm{SpN}$ and in $\mathrm{BbN}$ and repeated orally as many words as they could after each sentence was heard. Eight practice sentences were given prior to each noise condition. The sentences were delivered by a Matlab 2010a (Mathworks, USA) program and the experimenter scored participants' responses using a graphical user interface (GUI) not visible to participants. The SNR was varied adaptively to track the threshold for $50 \%$ words correct. The first sentence was always presented at a high SNR (8 dB and $10 \mathrm{~dB}$ for sentences in $\mathrm{SpN}$ and $\mathrm{BbN}$, respectively), and SNR was decreased by $4 \mathrm{~dB}$ on successive sentences until fewer than two words (i.e., $<50 \%$ correct) were recognized correctly. Then the SNR was increased or decreased by $2 \mathrm{~dB}$ on the next sentence when fewer $(<50 \%)$ or more than $(>50 \%)$ two words were recognized correctly. Participants were not given feedback about their performances. SRT was obtained by linear interpolation when two SNRs with the minimal distance (i.e., $2 \mathrm{~dB}$ ) had word scores that were higher and lower than $50 \%$.

\section{EEG experiment}

\section{Acoustic stimulus}

The stimulus was a 120 -ms-long /i/ syllable (Fig $\mathbf{2 A}$ ) with a falling $\mathrm{F}_{0}$ contour (160 to 
$110 \mathrm{~Hz})\left(\right.$ Fig 2B). This corresponded to the $4^{\text {th }}$ lexical tone in Mandarin. It was pronounced by a male native Mandarin speaker. Previous studies have used flat $\mathrm{F}_{0}$ contours (e.g., Aiken and Picton, 2008; Song et al., 2010). The syllable with a natural falling $\mathrm{F}_{0}$ contour controls for exposure, as such contours are not encountered in English monosyllables (Krishnan et al., 2005; Wong et al., 2007). Moreover, the $\mathrm{F}_{0}$ of the stimulus corresponds in range and direction (generally falling) to the $\mathrm{F}_{0} \mathrm{~s}$ of the target speakers in the $\mathrm{BKB}$ sentences employed in the SPIN task. The syllable had a relatively constant amplitude envelope except that $5 \mathrm{~ms}$ rising and falling cosine windows were applied at onset and offset to avoid transients. The formants occurred in frequency regions where the harmonics are and are not resolved (F1, F2 and F3 frequencies were approximately 280, 2400 and $3100 \mathrm{~Hz}$, see Fig 2A).
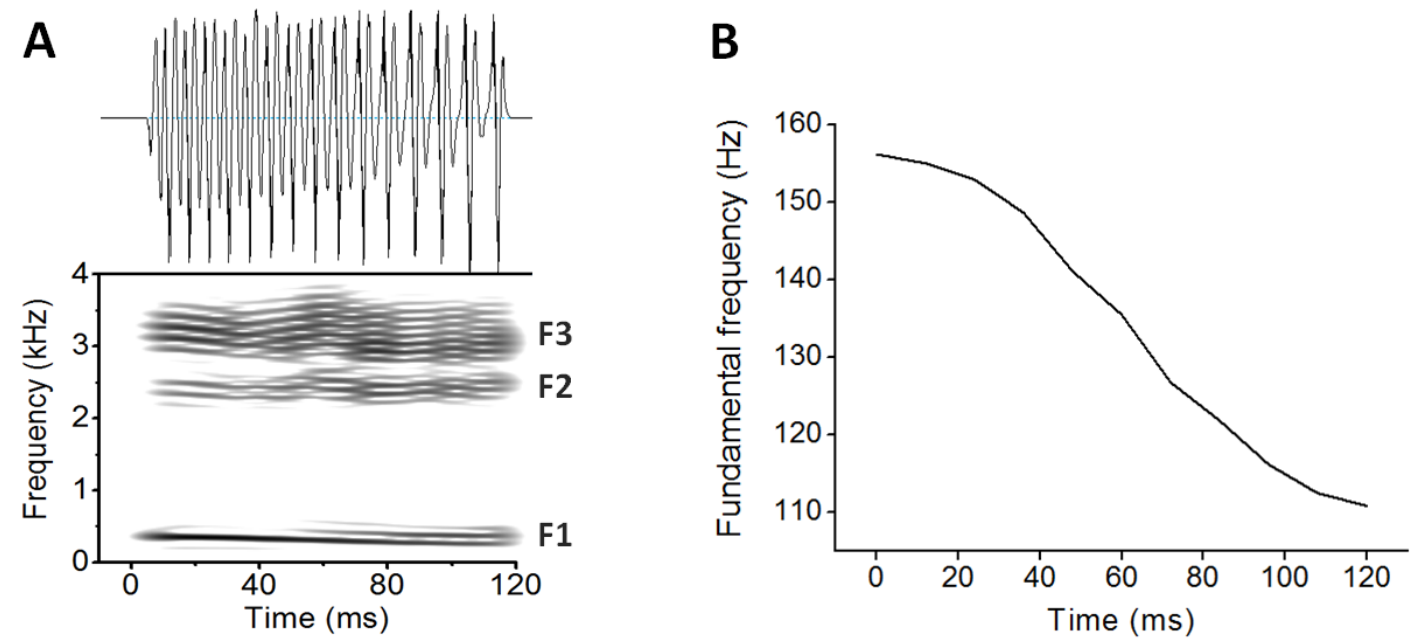

Figure 2. The syllable stimulus /i/ used during EEG recording. (A) The temporal waveform (top) and spectrogram (bottom) of the stimulus. F1, F2 and F3 frequencies are approximately 280, 2400 and $3100 \mathrm{~Hz}$, respectively. (B) $\mathrm{F}_{0}$ contour of the stimulus, which is a falling tone ranging between 160 and $110 \mathrm{~Hz}$, obtained by autocorrelation. The waveforms, spectrograms and $\mathrm{F}_{0}$ data were generated using PRAAT (Boersma and Weenink, 2013). 
The syllable was presented at the original polarity or negative polarity where the syllable was inverted. The stimuli were presented in three backgrounds: quiet ("no noise" or $\mathrm{NoN})$; steady-state speech-shaped noise $(\mathrm{SpN})$; and 16-speaker babble noise $(\mathrm{BbN})$. The last two corresponded to the types of noise in the SPIN task. The stimuli for NoN were presented at the same level to both groups of participants (see TABLE I) but the SNRs for SpN and $\mathrm{BbN}$ differed in level across Groups 1 and 2 (7 dB and -1dB respectively). There were 3200 sweeps at each polarity for each type of noise background (hence 6400 sweeps altogether). The 6400 sweeps were split into 16 segments for each background type and polarities were presented in a random order. Inter-stimulus intervals (ISI) varied randomly between 60 and $120 \mathrm{~ms}$ and syllables repetition rate was approximately five syllables per second.

\section{EEG recording procedure}

EEGs were recorded using an ActiveTwo system (Biosemi, The Netherlands) at a $16384 \mathrm{~Hz}$ sampling rate at the $\mathrm{Cz}$ (vertex), C3 and C4 position. The bilateral earlobes were used as references. Electrode impedance was always kept below $35 \mathrm{mV}$. Participants reclined comfortably in an adjustable chair located within an electromagnetic-shielded and sound-treated booth. The stimuli were presented via a Rogers LS3/5A loudspeaker (Falcon Acoustics, UK) positioned one meter from their ears at 0 degree horizontal azimuth directly in front of participants. The stimulus level (measured across time including inter-stimulus intervals) was 74.5 dB SPL at the 1 meter position before background noise was added in all the conditions. The stimulus levels after adding noise $(\mathrm{SpN}$ or $\mathrm{BbN})$ were $75.8 \mathrm{~dB}$ in Group 1 (SNR of $7 \mathrm{~dB}$ ) and $79.5 \mathrm{~dB}$ in Group 2 (SNR of $-1 \mathrm{~dB}$ ). Participants were instructed to close 
their eyes, relax and keep as still as possible to avoid movement artifacts. They were instructed not to attend actively to the stimuli and they were allowed to fall asleep to minimize discomfort because of protracted exposure to the repeated sounds. The entire in-booth test period lasted for approximately 70 minutes. They were monitored by the webcam throughout the test and no significant changes in head or body positions were observed. Algorithms that classified the sleep status in different periods were applied to the EEG data. These algorithms separated wakeful (conscious) from sleep (unconscious) states. Only the data from wakeful states were used in the current analyses (see Arousal states).

\section{Arousal states}

Wilf et al. (2016) reported cortical, but not subcortical, suppression whereas Portas et al. (2000) found suppression at both cortical and subcortical levels. Whilst this discrepancy needs resolving, both studios confirm that cortical activity is suppressed in sleep states. EEG data were only used from wakeful periods in the analyses in the present paper because significant correlations between SPIN performances and EEG parameters only occurred during wakeful periods (see Results). The detailed effects of sleep status on speech-evoked EEG responses is to be reported in the near future.

To separate wakeful from sleep periods, sleep spindles at the EEG sigma frequency (12 $\sim 16 \mathrm{~Hz}$ ) were located. These provide signatures about sleep (Warby et al., 2014) in Martin et al.'s (2013) method. Here EEGs at $\mathrm{Cz}$ were bandpass filtered into alpha ( $8 \sim 11 \mathrm{~Hz}$ ), sigma $(12 \sim 16 \mathrm{~Hz})$ and beta $(17 \sim 20 \mathrm{~Hz})$ bands using a 2nd-order zero-phase Butterworth filter. The filtered signals were then divided into 250-ms-long successive segments. A spindle was labeled when all three of the following conditions were met: 1) sigma root-mean-square 
(RMS) voltage in a given segment exceeded the threshold of the 95th percentile of the sigma RMS of all segments; 2) sigma RMS was higher than both alpha and beta RMS in the current segment; 3) two successive segments met both 1) and 2). 1) and 2) were invoked because sigma-band spectral domination is the major characteristic of sleep spindles (Martin et al., 2013; Warby et al., 2014). Extending requirements across two segments was included because sleep spindles usually last for at least 500 ms (De Gennaro and Ferrara, 2003).

The entire EEG recording was divided into 192 21-second-long epochs. Epochs were classified into three categories; wakefulness, sleep, and transition between wakefulness and sleep. Sleep epochs were those that contained at least one sleep spindle. Wakeful epochs were those that contained no spindles and were not adjacent to an epoch with a speech spindle. The remaining epochs were designated as transition epochs. Participants also gave a subjective rating of how much they slept after the test was completed (scale points from 1 to 7 , each of which had a textual description). There was a significant correlation between the subjective ratings and percentage of epochs classified algorithmically as 'sleep' $(p=0.002)$. This validated the classification algorithm.

\section{Frequency Following Responses (FFRs)}

FFRs were obtained from $\mathrm{Cz}$ (Skoe and Kraus, 2010). EEG signals were re-referenced to the average at the bilateral earlobes, bandpass filtered (70 4000 Hz) using a 2nd-order zero-phase Butterworth filter, and baseline corrected (based on the $50 \mathrm{~ms}$ pre-stimulus period in each sweep). To avoid movement artifacts, sweeps that had amplitudes exceeding $\pm 25 \mu \mathrm{V}$ were rejected (c.f., Song, Nicol and Kraus, 2011; Schoof and Rosen, 2016). FFRs with the original $\left(\mathrm{FFR}_{\text {orig }}\right)$ and negative $\left(\mathrm{FFR}_{\mathrm{neg}}\right)$ polarities were obtained by grand-averaging across 
sweeps with the respective polarities. FFRs that represent ENV (FFR $\mathrm{ENV}_{\text {) }}$ and TFS (FFR $\mathrm{TFS}_{\text {) }}$ were obtained by adding and subtracting FFR orig $_{\text {and }}$ FFR $_{\text {neg, }}$, respectively (Aiken and Picton,

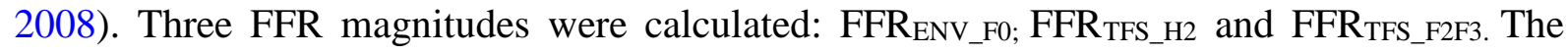
FFR $_{\text {ENV_F0 }}$ magnitude represents the neural magnitude of FFR ENV $_{\text {along }}$ the $F_{0}$ trajectory of

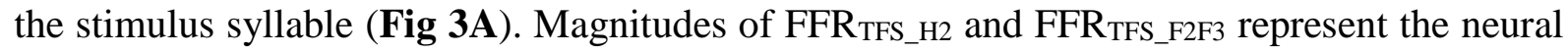
magnitudes of FFR ${ }_{\text {TFS }}$ along the resolved harmonics $H 2\left(2^{\text {nd }}\right.$ harmonics at $220 \sim 320 \mathrm{~Hz}$ representing F1) and unresolved F2 and F3 trajectories of the syllable respectively (Fig 3B). Magnitudes were calculated as follows.

A

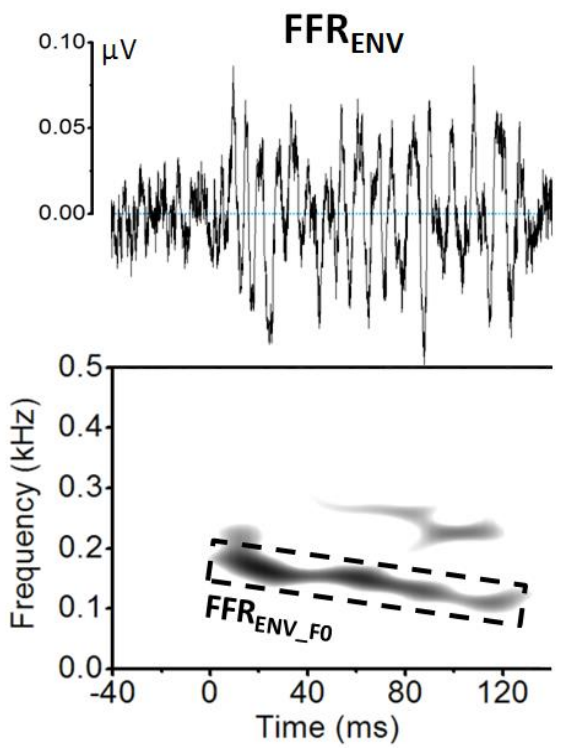

B

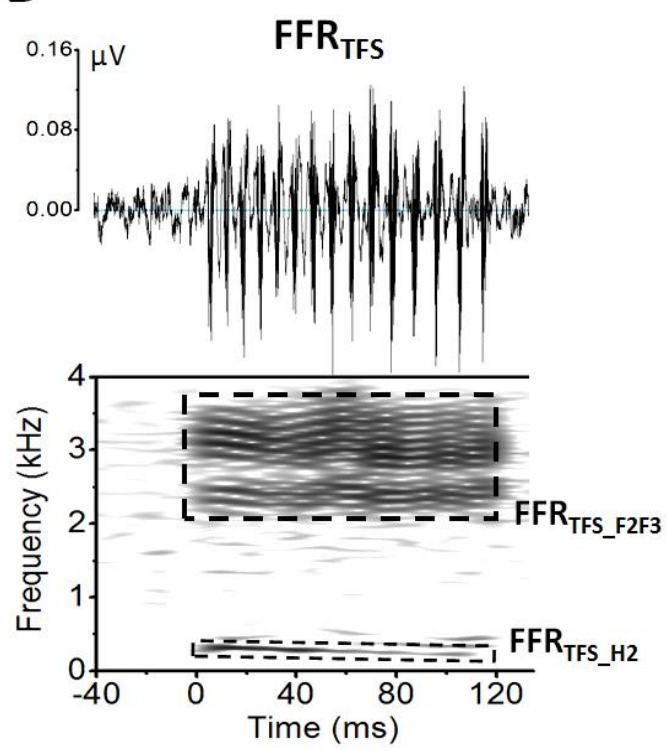

Figure 3. FFR $\mathrm{ENV}_{\mathrm{A}}$ (A) and $\mathrm{FFR}_{\mathrm{TFS}}$ (B) of waveforms (top) and spectrograms (bottom) averaged across 35 selected participants during wakeful periods in the quiet background (NoN) condition. The 35 participants were those whose numbers of sweeps were no fewer than 1,450 after artifact rejection during wakeful periods in NoN. For each participant, the waveforms were obtained based on randomly selecting wakeful epochs which contained 1,450 to 1,550 sweeps (see Normalization of sweep numbers). FFR ENV_F0 (at F0 range between $160 \sim 110 \mathrm{~Hz}$ ) 


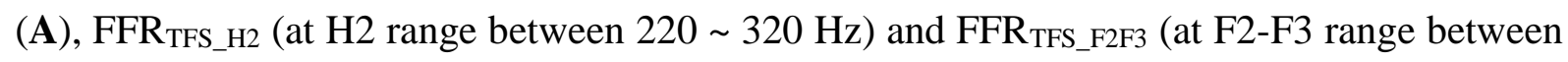
$2000 \sim 4000 \mathrm{~Hz}$ ) (B) are indicated by the dashed boxes. $\mathrm{X}$-axes give time in ms and '0' is the stimulus onset.

First, ENV-Fo, H2, F2 and F3 trajectories of the stimulus syllable were calculated. To obtain the ENV-F $\mathrm{F}_{0}$ trajectory, a set of $40 \mathrm{~ms}$ sliding windows (1-ms per step) was applied to the Hilbert envelope (Hilbert, 1912) of the syllable. Each $40 \mathrm{~ms}$ segment was Hanning-windowed, zero-padded to 1 second (to achieve $1 \mathrm{~Hz}$ frequency resolution) and Fourier-transformed. The frequency with the highest magnitude in the Fourier spectrum between 110 and $160 \mathrm{~Hz}$ ( $\mathrm{F}_{0}$ range of the syllable) was chosen as the $\mathrm{F}_{0}$ value at each step. $\mathrm{H} 2$, F2 and F3 trajectories were obtained in the same way, except that: 1) the sliding windows were applied to the syllable rather than the Hilbert envelope of the syllable; 2) the $\mathrm{H} 2$ value at each step was selected in the range of $\mathrm{H} 2(220 \sim 320 \mathrm{~Hz}) ; 3)$ instead of zero-padding and applying a Fourier transform, F2 and F3 values at each step were chosen based on the magnitudes of the spectral profile (via cepstral smoothing, Proakis and Manolakis, 2007) in the F2 and F3 ranges respectively $(2200 \sim 2600 \mathrm{~Hz}$ and $2800 \sim 3500 \mathrm{~Hz})$.

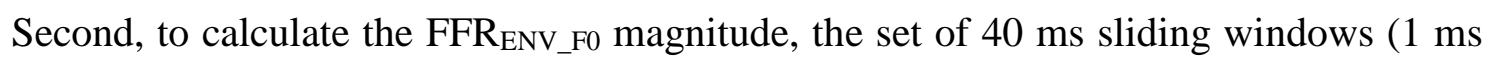

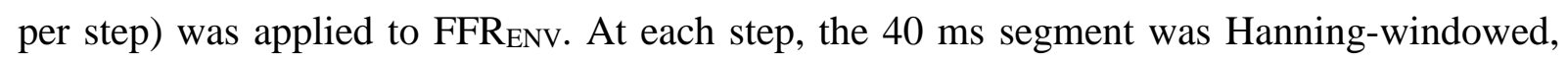
zero-padded to 1 second and Fourier-transformed. The mean log-magnitude (loge[power]) was measured across a $20 \mathrm{~Hz}$ bandwidth centered at the frequency of the ENV-F 0 trajectory of the syllable stimulus at that step. The magnitudes were then averaged across all steps covering the 
$120 \mathrm{~ms}$ syllable length. In addition, as FFR $\mathrm{ENV}_{\mathrm{N}}$ occurs at the auditory brainstem after a neural transmission time of between 5 and 10 ms (Chandrasekaran and Kraus, 2010; Skoe and Kraus,

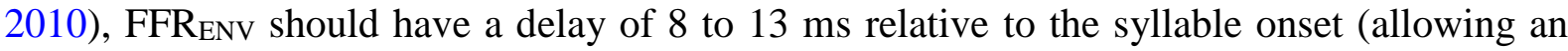
additional $3 \mathrm{~ms}$ for air-transmission from loudspeaker to cochlea). The final FFR ENV_F0 $_{2}$ magnitude was the maximum magnitude for a time lag between 8 and $13 \mathrm{~ms}$ ( $1 \mathrm{~ms}$ steps). Magnitudes of FFR $\mathrm{TFS}_{-} \mathrm{H} 2$ and $\mathrm{FFR}_{\mathrm{TFS} \_\mathrm{F} 2 \mathrm{~F} 3}$ were measured in the same way as $\mathrm{FFR}_{\mathrm{ENV} \_\mathrm{F} 0}$, except that: 1) the procedure was applied on the FFR

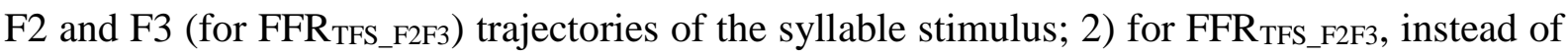
zero-padding and applying a Fourier transform, the magnitude at each step was the summed magnitude of the spectral profile (via cepstral smoothing) across a $150 \mathrm{~Hz}$ and $300 \mathrm{~Hz}$ bandwidth respectively centered at F2 and F3 of the syllable at that step; 3) as FFR early stages of auditory processing in the periphery (Aiken and Picton, 2008), the neural transmission time for $\mathrm{FFR}_{\mathrm{TFS} \_\mathrm{H} 2}$ and $\mathrm{FFR}_{\mathrm{TFS} \_\mathrm{F} 2 \mathrm{~F} 3}$ was set to $5 \mathrm{~ms}$ at maximum, resulting in time lags between 3 and $8 \mathrm{~ms}$ with the air-transmission time included.

\section{Cortical responses}

Electrodes C3 and C4 were used to represent activity in the auditory cortex (Carpenter and Shahin, 2013; Noguchi, Fujiwara and Hamano, 2015). Theta-band (4 $6 \mathrm{~Hz}$, corresponding to stimulus repetition rate of $\sim 5$ syllables per second) phase-locking values (PLVs) were measured. EEGs were decimated to $1024 \mathrm{~Hz}$, re-referenced to the average of bilateral earlobes and bandpass filtered $(4 \sim 6 \mathrm{~Hz})$ using a 2nd-order zero-phase Butterworth filter. Sweeps where amplitudes exceeded $\pm 15 \mu \mathrm{V}$ on either electrode were rejected. Due to the relatively narrow frequency range, the theta-band signal normally does not have 
excessively high amplitude and $>90 \%$ of the sweeps were retained in almost all participants (except for one participant who retained $>80 \%)$. PLV time series $(P L V(t))$ were calculated using the following formula (Morillon et al., 2012):

$$
P L V(t)=\left|\frac{1}{n} \sum_{i=1}^{n} \mathrm{e}^{j \phi_{i}(t)}\right|
$$

where $n$ denotes the total number of sweeps, $\phi_{i}(t)$ denotes the Hilbert phase series of the filtered EEG of the $i$ th sweep time-locked to the syllable onset. Hilbert phase was used as it locks to stimuli even when EEG amplitude variation occurs because of eye closure and relaxation (Thatcher, 2012). $P L V(t)$ were then averaged across the $120 \mathrm{~ms}$ syllable length. As neural transmission from cochlea to auditory cortex takes 10 to $15 \mathrm{~ms}$ in primates (Lakatos et al., 2007), the final PLV was taken as the maximum among values with time lags between 13 and $18 \mathrm{~ms}$ (1 ms steps) with the air-transmission time included.

Measurements of all EEG parameters (FFRs and PLV) were obtained using Matlab 2010a.

\section{Statistical analyses}

\section{Within-subject ANOVAs and linear regression analyses}

Within-subject Repeated Measures ANOVAs were conducted for the four EEG parameters (i.e., FFR $\mathrm{ENV}_{\text {_F0 }}, \mathrm{FFR}_{\mathrm{TFS} \_\mathrm{H} 2}, \mathrm{FFR}_{\mathrm{TFS} \_\mathrm{F} 2 \mathrm{F3}}$ and PLV) across the three background types (i.e., NoN, $\mathrm{SpN}$ and $\mathrm{BbN}$ ) for Groups 1 and 2, respectively. Post-hoc within-subject pairwise $t$-tests were conducted if the main effects of background type (with Greenhouse-Geisser correction) were significant in the ANOVA. 
Linear regression analyses were conducted for each group of participants. In each group, the analyses were conducted for all participants (the 'HA + non-HA' analysis) and for non-HA participants only (the 'non-HA only' analysis). In the 'HA + non-HA' analysis, SRT in SpN was predicted by the four EEG parameters (FFRENV_F0, FFR TFS_H2 $_{2}$ FFRTFS_F2F3 and PLV) obtained from the EEG recording whilst syllables were presented in SpN. Similarly, SRT in $\mathrm{BbN}$ was predicted by the four EEG parameters obtained from the EEG recording whilst syllables were presented in $\mathrm{BbN}$. A second regression analysis was further performed that excluded HA users (the 'non-HA only' analysis) to assess the effect of neural responses after controlling for audibility and hearing loss, as stimulus audibility information was not available for HA users in the present study. PTA (averaged across $0.25 \sim 4 \mathrm{kHz}$ and across left and right ears) was included as a predictor in addition to the EEG parameters to assess the effect of neural responses. A non-zero intercept was always included as an IV in each regression equation. Best-Subsets Regression was used, which chose predictors that generated the lowest Akaike information criterion (AIC). This provided the optimal model with best goodness of fit and the least chance of overfitting (Burnham and Anderson 2003). Multicollinearity was diagnosed if the variance inflation factors (VIFs) of any predictor were above 10 or if the condition index was above 30 (Hair. et al., 2010). The assumption that residuals were normally-distributed was violated if the Shapiro-Wilk test on the residuals had a $p$-value below 0.05 (Shapiro and Wilk, 1965). A subset was discarded when there was multicollinearity or normality of residuals was violated. Furthermore, outliers which had Cook's distances above 1 (Cook, 1979) were removed.

\section{Normalization of sweep numbers}


FFRs have small amplitudes, and averages of large numbers of stimulus repetitions are needed for reliable responses (Skoe and Kraus, 2010). Around 1,500 sweeps are needed after artifact rejection (c.f., Dajani et al., 2005; Wong et al., 2007). Participants' data on any particular background type were not included in subsequent analyses if the number of retained wakeful sweeps was below 1,450 for that background type.

Furthermore, since the current EEG parameters are based on phase-locked activities whose magnitudes are sensitive to the number of sweeps (Aviyente et al., 2011), problems can arise during statistical analyses if numbers of sweeps differ significantly across participants and background types. To reduce the risk of this happening, the number of sweeps was normalized to around 1,500 for each background type and participant. The normalization was conducted by randomly selecting the wakeful epochs which contained 1,450 to 1,550 artifact-free sweeps (summed across both polarities). The EEG parameters were then obtained from the selected epochs. This random selection procedure was repeated 100 times for each background type, giving 100 estimates of each EEG parameter. Measures averaged over the 100 estimates were used in the final statistical analyses. The process thus ensured that measurements of the EEG parameters were always based on around 1,500 sweeps regardless of the rejection rate. The normalization procedures for FFRs and PLV were conducted separately.

\section{Controlling for background EEG noise in FFRs}

As FFR magnitudes are small, their robustness can be influenced by the background EEG noise levels across participants (Skoe and Kraus, 2010). Therefore, the magnitudes of background EEG noises were obtained, and it was confirmed that magnitudes of FFR 
parameters were statistically above the noise level. We also tested whether EEG noise influenced the relationship between FFRs and the behavioral data by conducting partial correlation analyses between FFR parameters and SRTs after noise magnitudes were controlled for. The $50 \mathrm{~ms}$ pre-stimulus period was used as the background period. A set of 40 ms sliding windows (1 ms steps) was applied on the background period as described in the procedures for calculating $\mathrm{FFR}_{\mathrm{ENV}_{-} \mathrm{F} 0}, \mathrm{FFR}_{\mathrm{TFS} \_\mathrm{H} 2}$ and $\mathrm{FFR}_{\mathrm{TFS} \_\mathrm{F} 2 \mathrm{~F} 3}$ magnitudes. Each $40 \mathrm{~ms}$ segment was Hanning-windowed, zero-padded to 1 second and Fourier-transformed. For

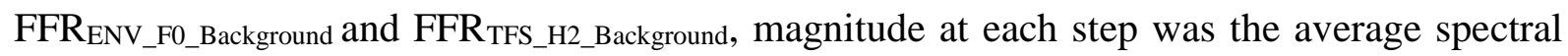
log-magnitude of FFR $\mathrm{ENV}_{\text {across }} 110$ and $\sim 160 \mathrm{~Hz}$ (range of $\mathrm{F}_{0}$ ) and that of FFR $\mathrm{TFS}_{\mathrm{TFS}}$ across $220 \sim 320 \mathrm{~Hz}$ (range of H2), respectively. For FFR TFS_F2F3_Background, instead of zero-padding and applying the Fourier transform, its magnitude at each step was calculated as the summed

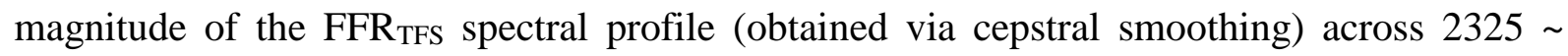
$2475 \mathrm{~Hz}(150 \mathrm{~Hz}$ bandwidth centered at $2400 \mathrm{~Hz}(\mathrm{~F} 2))$ and $2950 \sim 3250 \mathrm{~Hz}(300 \mathrm{~Hz}$ bandwidth centered at $3100 \mathrm{~Hz}(\mathrm{~F} 3)$ ). The final magnitudes were the averages across all steps of the background period. The procedure for normalization of sweep numbers for the noise magnitudes was the same as that done when the EEG parameters were calculated.

ANOVAs, t-tests, linear regressions and partial correlations were all conducted using SPSS 13.0 (SPSS Inc., USA).

\section{RESULTS}

\section{A. Behavioral results}


Fig 4 shows the SRT results across all 47 participants. Consistent with Rosen et al. (2013), SRT was significantly lower (i.e., better speech performance) in $\mathrm{SpN}$ (mean $\pm \mathrm{SE}$, $-4.13 \pm 0.21 \mathrm{~dB}$ ) than in $\mathrm{BbN}($ mean $\pm \mathrm{SE}, 0.54 \pm 0.22 \mathrm{~dB})$ (two-tailed $t$-tests, $\mathrm{t}_{(46)}=-30.127$, $\left.p<10^{-31}\right)$.

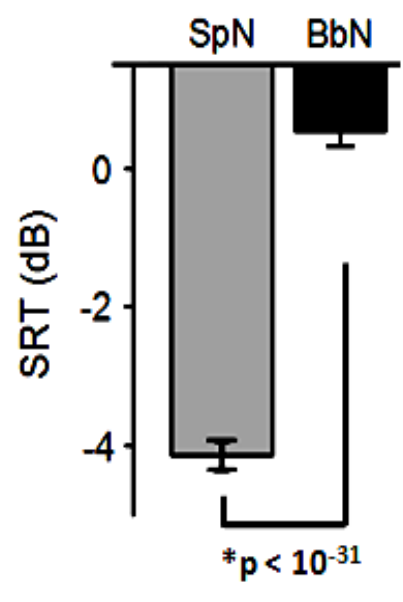

Figure 4. SRT results under $\mathrm{SpN}$ (grey) and $\mathrm{BbN}$ (black) across all 47 participants. The asterisk denotes $p<0.05$. Error bars denote SEs.

\section{B. Robustness of FFRs}

First, the magnitudes of the three FFR parameters (FFR $\mathrm{ENV}_{-} \mathrm{F} 0, \mathrm{FFR}_{\mathrm{TFS}} \mathrm{H} 2, \mathrm{FFR}_{\mathrm{TFS}}$ _F2F3) were compared with the magnitudes of their respective EEG background noise

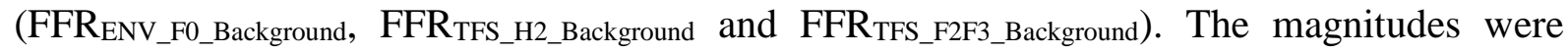
significantly higher for all three FFR parameters than their respective EEG background noise for all three background types (NoN, $\mathrm{SpN}$ and $\mathrm{BbN}$ ) in both groups of participants (two-tailed $t$-tests, all $p<0.001)$. This confirmed the robustness of FFRs at the group level. 


\section{Neural results across acoustic background types}

Next, within-subjects Repeated Measures ANOVAs were conducted for the four EEG parameters (three FFR parameters and PLV) across the background types separately for Groups 1 (SNR of $7 \mathrm{~dB}$ ) and 2 (SNR of $-1 \mathrm{~dB}$ ) (Fig 5). Only data from participants whose numbers of sweeps were above 1,450 for all three background types during wakeful periods were used. This resulted in 15 and 17 participants being retained in Groups 1 and 2, respectively, with 4 HA users in each group. Age (HA plus non-HA participants; Group 1 vs. 2: $\left.t_{(30)}=0.666, p=0.511\right)$ and audiometric threshold in non-HA participants (averaged across frequencies from 0.25 to $4 \mathrm{kHz}$ and across both ears; Group 1 vs. $2: t_{(22)}=-0.161, p=0.873$ ) did not differ between the two groups. Post-hoc pairwise t-tests (two-tailed, all with Bonferroni corrections) were conducted when a significant main effect (with Greenhouse-Geisser correction) of background type occurred in each analysis. A significant main effect was found for FFR $\mathrm{ENV}_{-} \mathrm{F0}$ in Group $2\left(F_{(2,14)}=15.615 ; p<0.001\right)$, with a

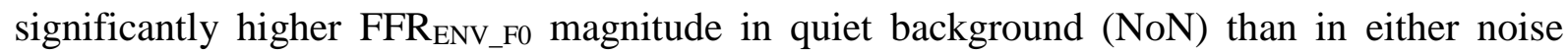

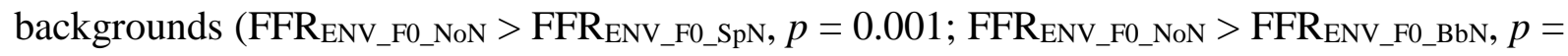
0.001). The difference in FFR $\mathrm{ENV}_{-} \mathrm{F} 0$ magnitude between $\mathrm{SpN}$ and $\mathrm{BbN}$ was not significant ( $p$ $=1.000)$. Significant main effects of background type were found for PLV in both groups

$\left(\right.$ Group 1: $\mathrm{F}_{(2,12)}=15.112, p<0.001 ;$ Group 2: $\left.F_{(2,14)}=19.548, p<10^{-4}\right)$. Post-hoc comparisons showed that, for Group 1, PLV was significantly higher in NoN and SpN than in $\mathrm{BbN}\left(\mathrm{PLV}_{\mathrm{NoN}}>\mathrm{PLV}_{\mathrm{BbN}}, p<0.001 ; \mathrm{PLV}_{\mathrm{SpN}}>\mathrm{PLV}_{\mathrm{BbN}}, p<0.001\right)$; for Group 2, PLV was significantly higher in NoN than in either noise background $\left(\mathrm{PLV}_{\mathrm{NoN}}>\mathrm{PLV}_{\mathrm{SpN}}, p=0.003\right.$; $\mathrm{PLV}_{\mathrm{NoN}}>\mathrm{PLV}_{\mathrm{BbN}}, p<0.001$ ), and PLV was significantly higher in $\mathrm{SpN}$ than in $\mathrm{BbN}$ 
$\left(\mathrm{PLV}_{\mathrm{SpN}}>\mathrm{PLV}_{\mathrm{BbN}}, p=0.023\right)$.

FFR $_{\text {ENV_F0 }} \quad$ FFR $_{\text {TFS_H2 }} \quad$ FFR $_{\text {TFS_F2F3 }} \quad$ PLV
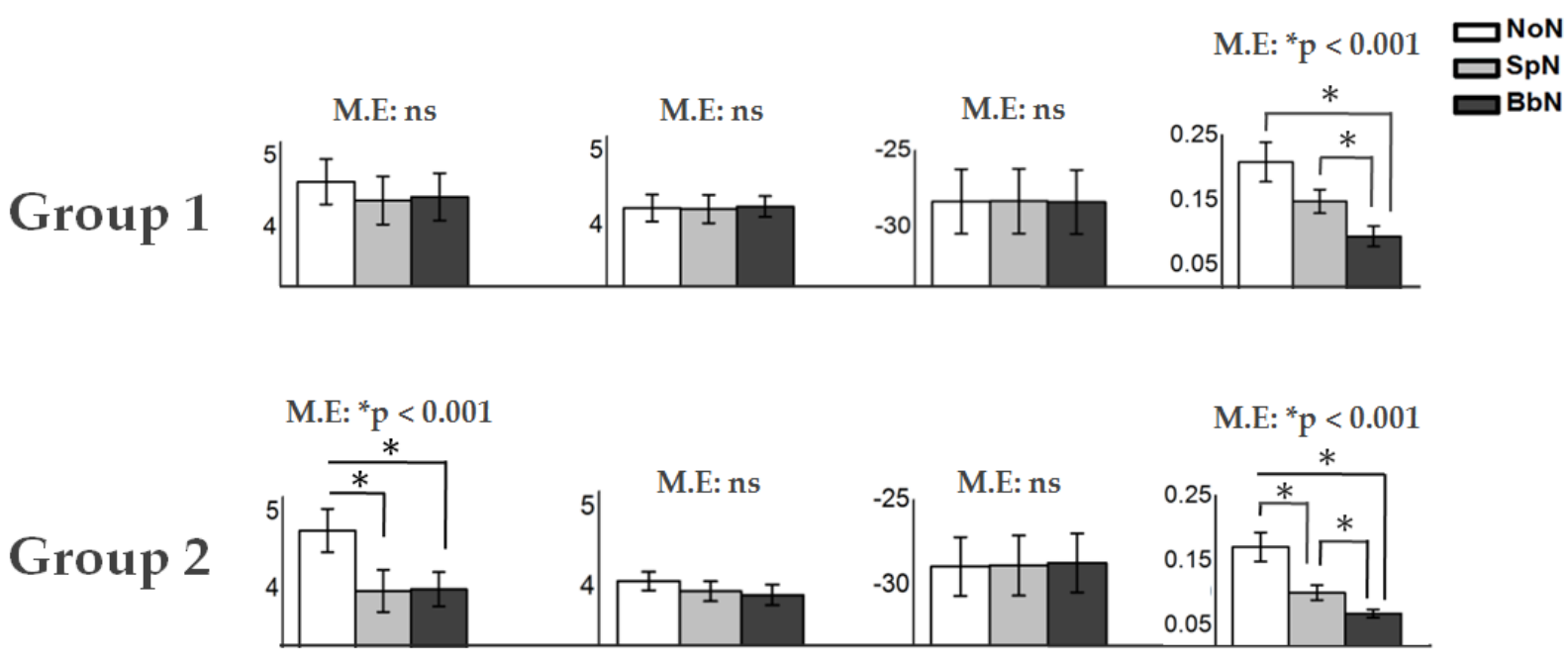

Figure 5. The four EEG parameters $\left(\mathrm{FFR}_{\mathrm{ENV} \_\mathrm{F} 0}, \mathrm{FFR}_{\mathrm{TFS} \_\mathrm{H} 2}, \mathrm{FFR}_{\mathrm{TFS} \_\mathrm{F} 2 \mathrm{F3}}\right.$ and PLV) under three background types (NoN, $\mathrm{SpN}$ and $\mathrm{BbN})$. Sections at the top and bottom give data from Groups 1 and 2, respectively. For FFRs, y-axes denote spectral log-magnitude (in dB). M.E.: main effects. ns: non-significant. Asterisks for the post-hoc pairwise t-tests denote $p<0.05$. Error bars denote SEs.

\section{Neural-behavioral relationship}

Linear regression was used to evaluate neural-behavioral relationships. For each group of participants, the four EEG measures were used to predict SRT in $\mathrm{SpN} / \mathrm{BbN}$ ('HA + non-HA'). PTA was also included as a predictor in a second analysis that excluded the HA users ('non-HA only'). According to the data-inclusion criterion (number of sweeps $\geq 1,450$ during wakeful periods), 18 (6) and 20 (6) participants were retained respectively in $\mathrm{SpN}$ and 
$\mathrm{BbN}$ for Group 1, and 19 (5) and 21 (5) participants were retained respectively in $\mathrm{SpN}$ and $\mathrm{BbN}$ for Group 2 (the number in brackets being the number of HA users).

Best-Subsets Regression results are summarized in TABLE II ('HA + non-HA') and TABLE III ('non-HA only'). For both 'HA + non-HA' and 'non-HA only' conditions, significant correlations between SRTs and EEG parameters were only found in Group 2 (SNR of $-1 \mathrm{~dB}$ during EEG recording). Specifically, for Group 2; i) in the 'HA + non-HA' condition, PLV correlated negatively with SRT in $\mathrm{SpN}\left(t_{(17)}=-2.265, p=0.037\right.$, see TABLE II and Fig

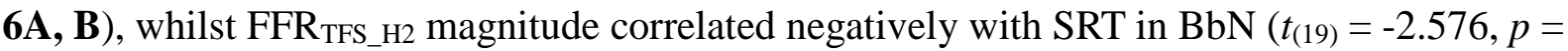
0.019, see TABLE II and Fig 6C, D); ii) in the 'non-HA only' condition, after controlling for PTA, FFRENV_Fo magnitude and PLV correlated negatively with SRT in SpN (FFRENV_F0: $t_{(10)}$ $=-2.246, p=0.049 ;$ PLV: $t_{(10)}=-2.450, p=0.034$; see TABLE III), whilst FFR ${ }_{\mathrm{TFS} \_\mathrm{H} 2}$ magnitude and PLV correlated negatively with SRT in BbN (FFR TFS_H2: $_{(12)}=-3.009, p=$ 0.011; PLV: $t_{(12)}=-8.256, p<10^{-5}$; see TABLE III).

TABLE II. Results of Best-Subsets linear regression for 'HA + non-HA' participants in Groups 1 and 2. SRTs (in $\mathrm{SpN} / \mathrm{BbN}$ ) were predicted by the four EEG parameters. $N$ denotes the number of participants based on the data inclusion criterion that the number of sweeps $\geq$ 1,450 during wakeful periods; numbers shown in brackets denote the number of HA participants. $R_{a}^{2}$ denotes the adjusted R-square of the models. Significant $p$ values are in bold and $*$ significant at $p<0.05$.

Group $\quad \mathrm{N} \quad \mathrm{R}_{\mathrm{a}}{ }^{2} \quad$ Noise type Dependent variable Predictor(s) T-value(s) p-value(s)




\begin{tabular}{|c|c|c|c|c|c|c|c|}
\hline \multirow[t]{2}{*}{ Group 1} & $18(6)$ & 0.010 & $\mathrm{SpN}$ & SRT & $\mathrm{FFR}_{\text {TFS_H2 }}$ & -1.086 & 0.293 \\
\hline & $20(6)$ & 0.015 & $\mathrm{BbN}$ & SRT & FFR & -1.134 & 0.272 \\
\hline \multirow[t]{2}{*}{ Group 2} & $19(5)$ & 0.187 & $\mathrm{SpN}$ & SRT & PLV & -2.265 & $0.037 *$ \\
\hline & $21(5)$ & 0.220 & $\mathrm{BbN}$ & SRT & $\mathrm{FFR}_{\mathrm{TFS} \_\mathrm{H} 2}$ & -2.576 & $0.019 *$ \\
\hline
\end{tabular}
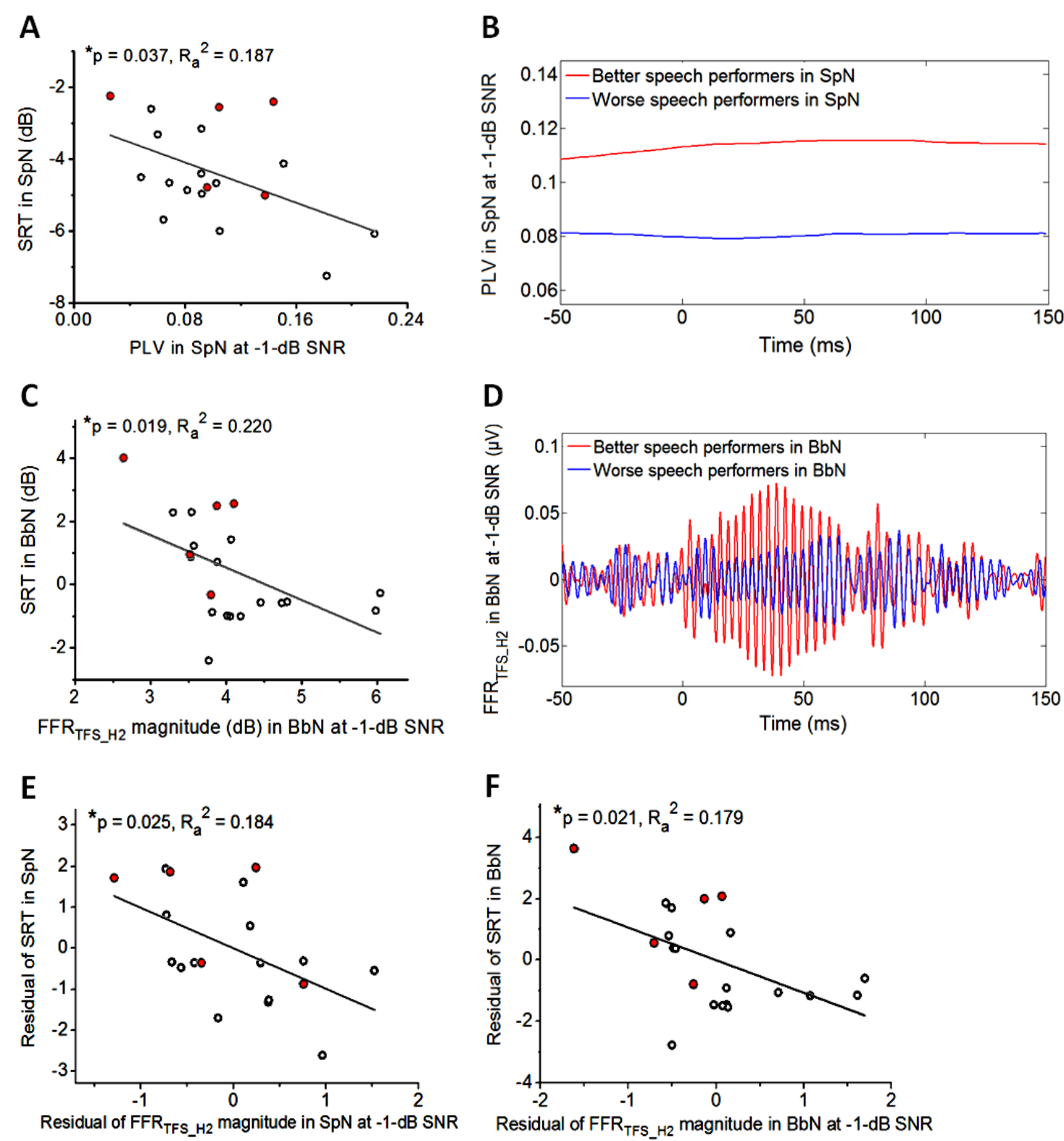

Figure 6. Neural-behavioral relation. Red dots represent HA participants. $p$ values and $R_{a}{ }^{2}$ are given. Asterisks denote $p<0.05$. Sections $\mathbf{A}$ and $\mathbf{C}$ give significant correlations between theta PLV and SRT in $\operatorname{SpN}(\mathbf{A})$ and between FFR TFS_H2 $_{2}$ magnitude and SRT in BbN (C) for Group 2 
according to the linear regression analyses (see TABLE II). Sections $\mathbf{B}$ and $\mathbf{D}$ give time series of theta PLV in SpN (B) and FFR TFS_H2 $_{2}\left(\mathrm{FFR}_{\mathrm{TFS}}\right.$ bandpass filtered at $\mathrm{H} 2$ range of $220 \sim 320$ $\mathrm{Hz}$ ) in $\mathrm{BbN}$ (D) for Group 2. Red and blue lines represent the averages of better (SRTs lower than the group median) and worse (SRTs higher than the group median) SPIN performers, respectively. The time series were obtained from the sweeps with normalized numbers $(1,450$ $\sim$ 1,550) during the wakeful periods (see Normalization of sweep numbers). X-axes give time in $\mathrm{ms}$ and '0' is the stimulus onset. Sections $\mathbf{E}$ and $\mathbf{F}$ give the partial correlations for Group 2

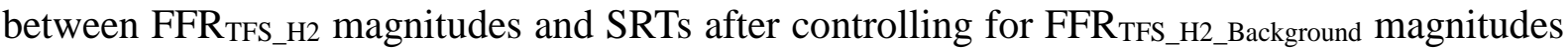
in $\mathrm{SpN}(\mathbf{E})$ and $\mathrm{BbN}(\mathbf{F})$. The $\mathrm{FFR}_{\mathrm{TFS} \_\mathrm{H} 2}$ residuals which were obtained from correlations

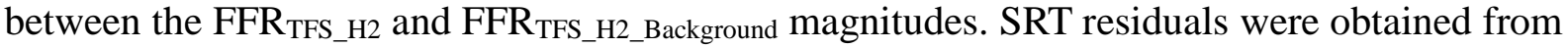
correlations between SRT and the FFRTFS_H2_Background magnitude.

TABLE III. Results of Best-Subsets linear regression for 'non-HA only' participants in Groups 1 and 2. SRTs (in $\mathrm{SpN} / \mathrm{BbN}$ ) were predicted by the four EEG parameters and PTA. $N$ denotes the number of participants based on the data inclusion criterion that the number of sweeps $\geq 1,450$ during wakeful periods. $R_{a}^{2}$ denotes the adjusted R-square of the models. Significant $p$ values $(p<0.05)$ are in bold and ${ }^{\wedge}=$ marginal significance $(p<0.1)$, * significant at $p<0.05$ and $* *=$ significant at $p<0.01$.

\begin{tabular}{|c|c|c|c|c|c|c|c|}
\hline Group & $\mathrm{N}$ & $\mathrm{R}_{\mathrm{a}}{ }^{2}$ & Noise type & Dependent variable & Predictor(s) & T-value(s) & P-value(s) \\
\hline \multirow[t]{2}{*}{ Group 1} & 12 & 0.114 & $\mathrm{SpN}$ & SRT & PTA & 1.555 & 0.151 \\
\hline & 14 & 0.246 & $\mathrm{BbN}$ & SRT & PTA & 2.289 & $0.041 *$ \\
\hline
\end{tabular}




\begin{tabular}{|c|c|c|c|c|c|c|c|}
\hline \multirow[t]{6}{*}{ Group 2} & 14 & 0.538 & $\mathrm{SpN}$ & SRT & $\mathrm{FFR}_{\text {ENV_F0 }}$ & -2.246 & 0.049* \\
\hline & & & & & PLV & -2.450 & $0.034 *$ \\
\hline & & & & & PTA & 2.062 & $0.066^{\wedge}$ \\
\hline & 16 & 0.882 & $\mathrm{BbN}$ & SRT & $\mathrm{FFR}_{\mathrm{TFS} \_\mathrm{H} 2}$ & -3.009 & 0.011* \\
\hline & & & & & PLV & -8.256 & $<10^{-5 * *}$ \\
\hline & & & & & PTA & 7.600 & $<10^{-5 * *}$ \\
\hline
\end{tabular}

Partial correlation analyses (two-tailed) were conducted next between the FFR parameters (FFR ENV_F0, FFR $_{\text {TFS_H2 }}$ and FFRTFS_F2F3) and SRTs while controlling for the magnitudes of background EEG noise. This partialled out the effects of EEG noise on the FFR-SRT relation mathematically. Results showed that:

1) For Group 1, there were no significant correlations between any of the FFRs parameters and SRTs in $\mathrm{SpN}$ or $\mathrm{BbN}$ after the respective EEG noise magnitudes were controlled for in the 'HA + non-HA' or 'non-HA only' condition (all $p>0.2$ ).

2) For Group 2:

i) In the ' $\mathrm{HA}+$ non-HA' condition, there were no significant correlations between FFR $_{\text {ENV_F0, FFR }}$ TFS_F2F3 magnitudes and SRTs after FFR ENV_F0_Background, FFR $_{\text {TFS_F2F3_Background }}$ magnitudes were controlled for in either $\mathrm{SpN}$ or $\mathrm{BbN}$ (all $p>0.7$ ). There were significant negative correlations between FFR TFS_H2 $_{-}$magnitude and SRT in $\operatorname{SpN}\left(t_{(16)}=-2.464, p=0.025\right.$, Fig 6E), and between $\mathrm{FFR}_{\mathrm{TFS} \_\mathrm{H} 2}$ magnitude and $\mathrm{SRT}$ in $\mathrm{BbN}\left(t_{(18)}=-2.525, p=0.021\right.$, Fig 6F) after $\mathrm{FFR}_{\mathrm{TFS} \_\mathrm{H} 2 \_ \text {Background }}$ magnitudes were controlled for. The latter replicated the previous linear regression result on Group 2 (see TABLE II and Fig 6C). 
ii) In the 'non-HA only' condition, there were no significant correlations between any of the FFRs parameters and $\mathrm{SRTs}$ in $\mathrm{SpN}$ or $\mathrm{BbN}$ after the respective EEG noise magnitudes were controlled for (all $p>0.09$ ). To further test the influence of EEG background noise on the previous Best-Subset Regression results in 'non-HA only' (see TABLE III), magnitudes of FFRENV_F0_Background and FFRTFS_H2_Background were used as additional predictors in the Best-Subset equations in $\mathrm{SpN}$ and $\mathrm{BbN}$ respectively. After adding FFR ENV_F0_Background as a predictor in SpN, correlations between FFR ENV_Fo and SRT and between PLV and SRT were not significant (FFR ENV_Fo $_{-}$and SRT: $t_{(9)}=-1.361, p=0.207$; PLV and SRT: $t_{(9)}=-2.240, p=$ 0.052; see TABLE IV). Thus, it cannot be entirely ruled out that, while controlling for PTA,

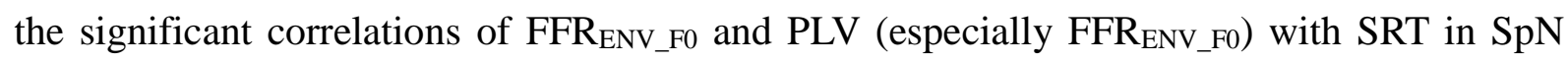
may be due to the influence of EEG background noise. Lack of significance may have occurred because of the small number of participants $(N=14)$ and the increased number of

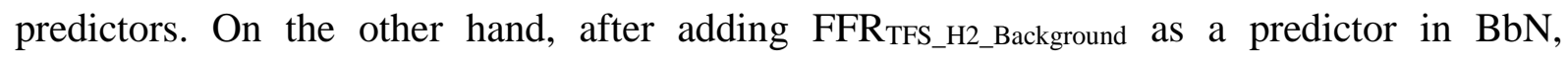
correlations between FFR $_{\text {TFS_H2 }}$ and SRT and between PLV and SRT were still significant $\left(\right.$ FFR $_{\text {TRS_H2 }}$ and SRT: $t_{(11)}=-2.548, p=0.027$; PLV and SRT: $t_{(11)}=-7.274, p<10^{-4}$; see TABLE IV), showing that the significant correlations were not influenced by the EEG background noise.

TABLE IV. Results of including magnitudes of EEG background noise as predictors for Group 2 ('non-HA only') participants based on the results of Best-subset linear regression. This analysis assesses the influence of the EEG background noise on the neural-behavioral relation. FFR ENV_F0_Background and FFR 
half of table) and in $\mathrm{BbN}$, (bottom half of table). $N$ denotes the number of participants based on the data inclusion criterion that the number of sweeps $\geq 1,450$ during wakeful periods. $R_{a}^{2}$ denotes the adjusted R-square of the models. Significant $p$ values $(p<0.05)$ are in bold and ${ }^{\wedge}$ $=$ marginal significance $(p<0.1), *=$ significant at $p<0.05$ and $* *=$ significant at $p<0.01$.

\begin{tabular}{|c|c|c|c|c|c|c|c|}
\hline Group & $\mathrm{N}$ & $\mathrm{R}_{\mathrm{a}}^{2}$ & Noise type & Dependent variable & Predictor(s) & T-value(s) & P-value(s) \\
\hline \multirow[t]{8}{*}{ Group 2} & 14 & 0.487 & $\mathrm{SpN}$ & SRT & $\mathrm{FFR}_{\text {ENV_F0 }}$ & -1.361 & 0.207 \\
\hline & & & & & PLV & -2.240 & $0.052^{\wedge}$ \\
\hline & & & & & PTA & 1.948 & $0.083^{\wedge}$ \\
\hline & & & & & FFRENV_F0_Background $_{\text {EN }}$ & -0.098 & 0.924 \\
\hline & 16 & 0.871 & $\mathrm{BbN}$ & SRT & FFR & -2.548 & $0.027 *$ \\
\hline & & & & & PLV & -7.274 & $<10^{-4 * *}$ \\
\hline & & & & & PTA & 6.170 & $<10^{-4 * *}$ \\
\hline & & & & & $\mathrm{FFR}_{\mathrm{TFS} \_\mathrm{H} 2 \_B a c k g r o u n d}$ & 0.197 & 0.847 \\
\hline
\end{tabular}

\section{DISCUSSION}

The present results support our hypothesis that SPIN perception is associated statistically with phase-locked neural encoding of Slow-rate ENV and TFS, rather than that of $\mathrm{F}_{0}$-rate ENV, in older adults who presented with different audiometric profiles. Magnitude of FFR $_{\text {ENV_Fo that }}$ reflects encoding of $\mathrm{F}_{0}$-rate ENV correlated significantly with behavioral performances in $\mathrm{SpN}$ when audiometric threshold was mathematically controlled for. The participants here showed wide individual variability of audiometric thresholds as is typical in aging populations (Gopinath et al., 2009; Humes et al., 2010) and hence should be more 
representative of clinical populations than previous studies (Anderson et al., 2011; Presacco et al., 2016; Schoof and Rosen, 2016). Some HA users were also included in the present study and contributed to the current results (the 'HA + non-HA' condition). This extended individual variability in the regression analyses (see Fig. 6; HA users are shown as red dots). However, it should be noted that stimulus audibility was not quantified for this group, and the numbers are small. Future work should allow more informative hearing aid-related analyses. Significant correlations between EEG parameters and SRT performance were found in Group 2 but not in Group 1. This indicated that individual variability of SPIN abilities may be better explained using EEG parameters obtained under low SNR, which is relatively challenging for speech perception (-1 dB in Group 2), compared to high SNR at which speech is fairly recognizable (7 $\mathrm{dB}$ in Group 1). The neural-behavioral relationship is summarized schematically in Fig 7 and is discussed further below.

Type of noise

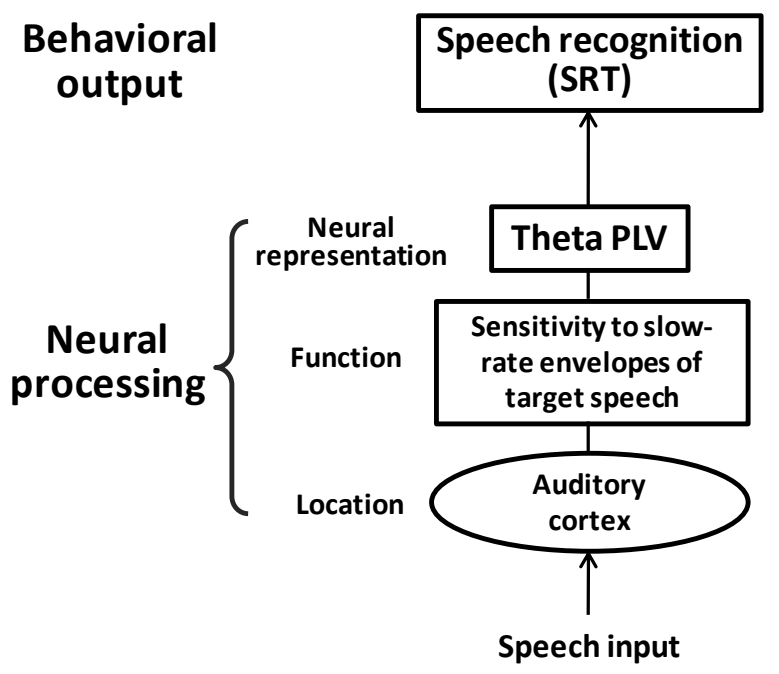

SpN \& BbN

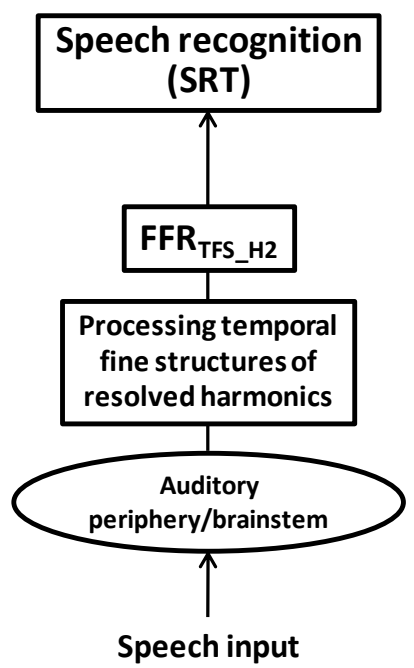

Figure 7. Summary of the neural-behavioral relationship in older adults who varied in audiometric hearing (the 'HA + non-HA' condition). Theta PLV, which reflects cortical 
sensitivity to Slow-rate ENV of target speech, was associated with speech performances in SpN. FFR TFS_H2 $_{2}$ magnitude, which reflects peripheral/brainstem processing of TFS in the resolved harmonics region, was associated with speech performances for both $\mathrm{SpN}$ and $\mathrm{BbN}$. Also see TABLE II and Figure 6.

\section{A. Cortical sensitivity to Slow-rate ENV}

The SRT result showed that speech perception was significantly better in $\mathrm{SpN}$ than in BbN (Fig 4), as reported in previous work (Rosen et al., 2013). Correspondingly, the neural results showed that theta-band PLV time-locked to the syllable onset was significantly higher in $\mathrm{SpN}$ than in $\mathrm{BbN}$ for both groups of participants and there were no significant differences for other EEG parameters between the two noise types (Fig 5). Also, PLV was higher in quiet than in noisy backgrounds (particularly in Group 2, see Fig 5). Such results may explain how speech perception deteriorates in noisy environments and why it is better in $\mathrm{SpN}$ than in $\mathrm{BbN}$. Previous research has reported that the theta PLV reflects neural sensitivity to the Slow-rate ENV in the auditory cortex (Luo and Poeppel, 2007; Howard and Poeppel, 2010) and that Slow-rate ENV is critical for speech perception (Drullman et al., 1994; Shannon et al., 1995; Arai et al., 1999). Furthermore, theta PLV obtained from scalp-EEG time-locked to input acoustic stimuli could reflect neural firing in the auditory cortex when decoding stimuli $(\mathrm{Ng}$, Logothetis and Kayser, 2013). SpN is a steady-state energetic masker (EM) with no linguistic information, whilst $\mathrm{BbN}$ is an $\mathrm{EM}$ with envelope modulations more similar to speech compared to $\mathrm{SpN}$. Also, $\mathrm{BbN}$ is an informational masker (IM) that can lead to phonetic 
interference (Rosen et al., 2013). BbN had no lexical interference in the present study because of the high number of speakers (16) in the babble (Hoen et al., 2007). It is likely that the combined EM and IM properties of BbN caused lower cortical sensitivity to Slow-rate ENV of the target speech, leading to worse SPIN performance than that in SpN. This differential cortical sensitivity may serve as the neural mechanism of SPIN performance which varies as a function of noise type. Moreover, the linear regression results from Group 2 showing that higher theta PLV was associated with better speech perception in SpN (see TABLE II and TABLE III) and BbN (after controlling for PTA in non-HA participants, see TABLE III) further highlighted the important role of cortical sensitivity to Slow-rate ENV during SPIN perception.

\section{B. Neural encoding of TFS in the resolved harmonics region}

The neural-behavioral relationship observed in Group 2 showed that higher FFR TFS_H2 $_{\text {H }}$ magnitude, which reflects phase-locked neural encoding of TFS in the resolved harmonics (H2) region, was associated with better speech perception in both $\mathrm{SpN}$ and $\mathrm{BbN}$ (TABLE II

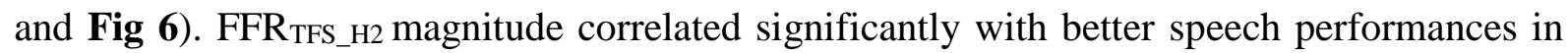
BbN after PTA was controlled for (TABLE III and IV). Previous studies using cochlear implant simulation methods have shown that encoding TFS is critical for SPIN perception (Zeng et al., 2005; Stickney et al., 2007; Eaves et al., 2011). Unprocessed (where TFSs were retained compared to those "processed" without TFS) low-frequency $(<500 \mathrm{~Hz})$ speech components benefited speech perception for both normal-hearing people and cochlear implant users (Turner et al., 2004; Qin and Oxenham, 2006; Carroll, Tiaden, and Zeng, 2011). A harmonic sieve, in which pitch of the target voice is extracted and used to segregate this voice 
from background noise, is a possible explanation (Bregman, 1994; Bird and Darwin, 1998; Binns and Culling, 2005). Pitch perception mainly relies on TFS (Smith et al., 2002; Zeng et al., 2004), especially TFS in the low-frequency resolved harmonics region (Oxenham, Micheyl and Keeble, 2009). In the present study, the syllable stimulus used during EEG recording had an $\mathrm{F}_{0}$ range and contour (falling) similar to those of the target voices in the sentence stimuli during the SPIN perception task. Therefore, better FFR TFS_H2 $_{2}$ representation reflects better processing of pitch information of the target speech, hence better voice segregation ability during SPIN perception.

\section{Neural encoding of $\mathbf{F}_{0}$-rate ENV}

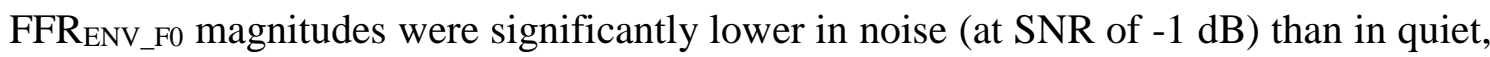
as reported in previous studies (Russo et al., 2004; Presacco, Simon and Anderson, 2016). This indicates how background noise affects speech perception. Anderson et al. (2011) showed that FFRENV_F0 magnitude correlated significantly with better SPIN performances in older adults. While only normal-hearing older adults (hearing thresholds $\leq 25 \mathrm{~dB} \mathrm{HL}$ at $\leq$ $4000 \mathrm{~Hz}$ ) were tested in Anderson et al. (2011), the present study recruited participants who varied in audiometric profiles (including some who wore HAs), which gives a more appropriate audiometric distribution for aging populations (Gopinath et al., 2009; Humes et al., 2010). The present results did not show any significant correlation between FFR ENV_F0 $_{2}$ magnitude and SPIN performance until PTA was mathematically controlled for in non-HA participants (for $\mathrm{SpN}$ in Group 2, see TABLE III). This significant correlation could also have resulted from the influence of EEG pre-stimulus magnitudes (TABLE IV), although such influence may simply reflected the variation in pre-stimulus brain activities and, hence, 
may need to be interpreted with caution. The correlation between FFRENV_Fo magnitude and SPIN performance indicates that phase-locked neural encoding of $F_{0}$-rate ENV may be associated with SRT only when participants have similar audiometric thresholds and such

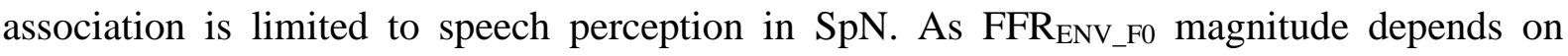
participants' sensitivity to the syllable, with poorer audibility leading to smaller FFRENV_F0 magnitude (Ananthakrishnan et al., 2016), higher degrees of hearing loss should cause smaller FFRENV_Fo magnitude. However, other evidence has shown that hearing loss can lead to greater encoding of $\mathrm{F}_{0}$-rate ENV compared to normal-hearing in animal studies (Kale and Heinz, 2010; Henry et al., 2014; Zhong et al., 2014) as well as in older human participants (Anderson et al., 2013). It has been suggested that such exaggerated encoding of $\mathrm{F}_{0}$-rate ENV distracts encoding of salient acoustic cues (e.g., TFS) in noisy environment and hence could be detrimental to SPIN perception (Henry et al., 2014; Zhong et al., 2014). Therefore, the extent of audiometric hearing loss should be controlled carefully when studying the relationship between SPIN performances and neural encoding of $\mathrm{F}_{0}$-rate ENV in older adults.

\section{Clinical implications}

Older adults who varied in their audiometric thresholds were tested in the present study. Audiometric impairments in older adults often manifest as high-frequency hearing loss (Humes et al., 2010). However, high-frequency amplification in older adults has limited clinical benefits for speech perception (Turner and Cummings, 1999; Ching et al., 2001; Horwitz, Ahlstrom and Dubno, 2008). People using auditory prostheses, such as hearing aids (HAs) have difficulty in understanding conversations in noise partly because of limited improvements in SNR despite advanced contemporary noise reduction approaches (Bentler, 
2005; Géléoc and Holt, 2014; Tremblay and Miller, 2014). Furthermore, even older adults with normal hearing experience difficulties in SPIN perception, especially in multi-speaker backgrounds, compared to normal-hearing young adults (Helfer and Freyman, 2008; Rajan and Cainer, 2008; Schoof and Rosen, 2014). Therefore, as well as developing strategies for restoring peripheral hearing and applying noise reduction approaches, it could be helpful to attend to neural biomarkers in the auditory pathway in order to determine optimal rehabilitation in older adults. In addition to decline in audiometric hearing, older adults have been shown to have diminished auditory processing of TFS that is related to poorer SPIN perception (Lorenzi et al., 2006; Grose and Mamo, 2010; Hopkins et al., 2008; Hopkins and Moore, 2011). This may be related to the loss of neural inhibition in the aging auditory system which is important for phase-locking to TFS information (Anderson et al., 2012). Furthermore, broadened frequency tuning at the auditory periphery due to hearing loss may decrease the phase-locking abilities to TFS causing SPIN perception to deteriorate further (Henry and Heinz, 2012). This echoes the present finding that peripheral/brainstem processing of TFS in the low-frequency resolved harmonics region is critical for SPIN perception. The present findings thus provide neural evidence to support signal processing strategies for cochlear implants that retain TFS information in the low-order harmonics region that render significant benefits during speech perception (Turner et al., 2004; Qin and Oxenham, 2006; Carroll et al., 2011).

It has been proposed that scalp-recorded electrophysiological measures, such as cortical auditory evoked potentials (cAEPs) (Tremblay and Miller, 2014) and speech-evoked FFRs (Dajani, Heffernan and Giguere, 2013; Bellier et al., 2015), could be used to evaluate the 
effects of HA fitting. Components of cAEPs (such as the N1-P2 complex) have been reported to be good indicators for assessing HA effects both in quiet and in background noise (Tremblay and Miller, 2014; Kuruvilla-Mathew, Purdy and Welch, 2015). While low-frequency (delta, theta and alpha) PLVs can predict both amplitudes and latencies of N1 and P2 in cAEPs in noisy backgrounds (Koerner and Zhang, 2015), it is plausible that PLV can also serve as a marker that helps to assess the effects of HA fitting on cortical encoding during SPIN perception. Bellier et al. (2015) demonstrated that magnitudes of speech-evoked FFR $_{\text {ENV }}$ and FFR TFS $_{\text {can }}$ be modulated by amending HA settings which generate different auditory stimulations. FFRs could thus be used as potential peripheral/subcortical markers that would benefit HA users. Therefore, a further clinical implication of the present study is that it could provide cortical and peripheral/subcortical neural markers for evaluating and optimizing the settings in HAs in older adults. Compared to evaluation of these neural markers, evaluation of SPIN performances tends to be a more direct approach to assess HA benefit. However, improvements of SPIN perception based on different HA settings may also be influenced by higher-level cognitive factors, such as working memory capacity (see a review by Lunner, Rudner and Rönnberg, 2009). Evaluation of sensory-neural markers in HAs could thus dissociate its role from individual cognitive factors in assisting SPIN perception. To this end, future work is needed to assess the neural-behavioral relation with further control of cognitive factors during SPIN perception.

\section{Acknowledgements}

This study was approved by the UCL Research Ethics Committee. The authors would 
like to thank Mr. Andrew Clark for his technical support of experimental setup and Dr. Tim Green for sharing the SRT test programs in Matlab.

\section{REFERENCES}

Ahissar, E., Nagarajan, S., Ahissar, M., Protopapas, A., Mahncke, H., and Merzenich, M.M. (2001). "Speech comprehension is correlated with temporal response patterns recorded from auditory cortex," Proc. Natl. Acad. Sci. 98, 13367-13372.

Aiken, S. J., and Picton, T. W. (2008). "Envelope and spectral frequency-following responses to vowel sounds," Hear. Res. 245, 35-47.

Ananthakrishnan, S., Krishnan, A., and Bartlett, E. (2016). "Human frequency following response: Neural representation of envelope and temporal fine structure in listeners with normal hearing and sensorineural hearing loss," Ear Hear. 37, 91-103.

Anderson, S., Parbery-Clark, A., Yi, H. G., and Kraus, N. (2011). "A neural basis of speech-in-noise perception in older adults," Ear Hear. 32, 750-757.

Anderson, S., Parbery-Clark, A., White-Schwoch, T., Drehobl, S., and Kraus, N. (2013). "Effects of hearing loss on the subcortical representation of speech cues," J. Acoust. Soc. Am. 133, 3030-3038.

Anderson, S., Parbery-Clark, A., White-Schwoch, T., and Kraus, N. (2012). "Aging affects neural precision of speech encoding," J. Neurosci. 32, 14156-14164.

Arai, T., Pavel, M., Hermansky, H., and Avendano, C. (1999). "Syllable intelligibility for temporally filtered LPC cepstral trajectories,” J. Acoust. Soc. Am. 105, 2783-2791.

Aviyente, S., Bernat, E. M., Evans, W. S., and Sponheim S. R. (2011). "A phase synchrony 
measure for quantifying dynamic functional integration in the brain," Hum. Brain Mapp. $32,80-93$.

Bellier, L., Veuillet, E., Vesson, J. F., Bouchet, P., Caclin, A., and Thai-Van, H. (2015). “Speech Auditory Brainstem Response through hearing aid stimulation,” Hear. Res. 325, 49-54.

Bench, J., Kowal, A., and Bamford, J. (1979). “The BKB (Bamford-Kowal-Bench) sentence lists for partially-hearing children,” Brit. J. Audiol. 13, 108-112.

Bentler, R. A. (2005). "Effectiveness of directional microphones and noise reduction schemes in hearing aids: A systematic review of the evidence," J. Am. Acad. Audiol. 16, 473-484.

Binns, C., and Culling, J. F. (2005). "The role of fundamental frequency (F0) contours in the perception of speech against interfering speech,” J. Acoust. Soc. Am. 117, 2606-2607.

Bird, J., and Darwin, C. J. (1998). "Effects of a difference in fundamental frequency in separating two sentences," in Psychophysical and Physiological Advances in Hearing, edited by A. R. Palmer, A. Rees, A. Q. Summerfield and R. Meddis (Whurr, London), pp. 263-269.

Boersma, P., and Weenink, D. (2013). Praat: doing phonetics by computer [Computer program] Version 5.3.51, Retrieved 2 June 2013.

Bregman, A. S. (1994). Auditory scene analysis: The perceptual organization of sound (MIT press).

Burnham, K. P., and Anderson, D. (2003). Model selection and multi-model inference: A Practical informatio-theoretic approach (Springer Science and Business Media).

Carpenter, A. L., and Shahin, A. J. (2013). "Development of the N1-P2 auditory evoked 
response to amplitude rise time and rate of formant transition of speech sounds," Neurosci. Lett. 544, 56-61.

Carroll, J., Tiaden, S., and Zeng, F. G. (2011) "Fundamental frequency is critical to speech perception in noise in combined acoustic and electric hearing," J. Acoust. Soc. Am. 130, 2054-2062.

Chandrasekaran, B., and Kraus, N. (2010). "The scalp-recorded brainstem response to speech: Neural origins and plasticity," Psychophysiology 47, 236-246.

Ching, T. Y., Dillon, H., Katsch, R., and Byrne, D. (2001). "Maximizing effective audibility in hearing aid fitting," Ear Hear. 22, 212-224.

Cook, R. D. (1979). "Influential observations in linear regression,” J. Am Stat. Assoc. 74, 169-174.

Dajani, H. R., Purcell, D., Wong, W., Kunov, H., and Picton, T. W. (2005). "Recording human evoked potentials that follow the pitch contour of a natural vowel," IEEE Trans. Biomed. Engineer. 52, 1614-1618.

Dajani, H. R., Heffernan, B. P., and Giguere, C. (2013). "Improving hearing aid fitting using the speech-evoked auditory brainstem response," $35^{\text {th }}$ Ann. Int. Conf. IEEE pp.2812-2815.

De Gennaro, L., and Ferrara, M. (2003). “Sleep spindles: An overview," Sleep Med. Rev. 7, 423-440.

Doelling, K.B., Arnal, L.H., Ghitza, O., and Poeppel, D. (2014). “Acoustic landmarks drive delta-theta oscillations to enable speech comprehension by facilitating perceptual parsing," Neuroimage, 85, 761-768.

Drullman, R., Festen, J. M., and Plomp, R. (1994). “Effect of temporal envelope smearing on 
speech reception,”J. Acoust. Soc. Am. 95, 1053-1064.

Eaves, J. M., Summerfield, A. Q., and Kitterick, P. T. (2011). "Benefit of temporal fine structure to speech perception in noise measured with controlled temporal envelopes," J. Acoust. Soc. Am. 130, 501-507.

Faulkner, A., Rosen, S., and Smith, C. (2000). "Effects of the salience of pitch and periodicity information on the intelligibility of four-channel vocoded speech: Implications for cochlear implants," J. Acoust. Soc. Am. 108, 1877-1887.

Fujihira, H., and Shiraishi, K. (2015). "Correlations between word intelligibility under reverberation and speech auditory brainstem responses in elderly listeners," Clin. Neurophysiol. 126, 96-102.

Géléoc, G. S., and Holt, J. R. (2014). "Sound strategies for hearing restoration,” Science 344, 1241062.

Gopinath, B., Rochtchina, E., Wang, J. J., Schneider, J., Leeder, S. R., and Mitchell, P. (2009). "Prevalence of age-related hearing loss in older adults: Blue Mountains Study," Arch. Intern. Med. 169, 415-418.

Greenberg, S., Carvey, H., Hitchcock, L., and Chang, S. (2003). "Temporal properties of spontaneous speech - A syllable-centric perspective,” J. Phonet. 31, 465-485.

Grose, J. H., and Mamo, S. K. (2010). "Processing of temporal fine structure as a function of age,” Ear Hear. 31, 755.

Hair, J. F., Anderson, R. E., Babin, B. J., and Black, W. C. (2010). Multivariate data analysis: A global perspective (Upper Saddle River, NJ Pearson).

Helfer, K. S., and Freyman, R. L. (2008). “Aging and speech-on-speech masking,” Ear Hear. 
29, 87-98.

Henry, K.S., and Heinz, M.G. (2012). "Diminished temporal coding with sensorineural hearing loss emerges in background noise," Nat. Neurosci. 15, 1362-1364.

Henry, K.S., Kale, S., and Heinz, M.G. (2014). "Noise-induced hearing loss increases the temporal precision of complex envelope coding by auditory-nerve fibers," Front. Syst. Neurosci. 8:20.

Hilbert, D. (1912). "Begründung der kinetischen Gastheorie,” Mathematische Annalen 72, $562-577$.

Hoen, M., Meunier, F., Grataloup, C. L., Pellegrino, F., Grimault, N., Perrin, F., Perrot, X., and Collet, L. (2007). "Phonetic and lexical interferences in informational masking during speech-in-speech comprehension," Speech Comm. 49, 905-916.

Hopkins, K., Moore, B. C., and Stone, M. A. (2008). "Effects of moderate cochlear and hearing loss on the ability to benefit from temporal fine structure information in speech," J. Acoust. Soc. Am. 123, 1140-53.

Hopkins, K., and Moore, B. C. (2011). "The effects of age and cochlear hearing loss on temporal fine structure sensitivity, frequency selectivity, and speech reception in noise," J. Acoust. Soc. Am. 130, 334-49.

Horwitz, A. R., Ahlstrom, J. B., and Dubno, J. R. (2008). "Factors affecting the benefits of high-frequency amplification,” J. Speech, Lang. Hear. Res. 51, 798-813.

Howard, M. F., and Poeppel, D. (2010). “Discrimination of speech stimuli based on neuronal response phase patterns depends on acoustics but not comprehension,” J. Neurophysiol. $104,2500-2511$. 
Humes, L. E., Kewley-Port, D., Fogerty, D., and Kinney, D. (2010). "Measures of hearing threshold and temporal processing across the adult lifespan," Hear. Res. 264, 30-40.

Kale, S., and Heinz, M.G. (2010). "Envelope coding in auditory nerve fibers following noise-induced hearing loss,” J. Assoc. Res. Otolaryngol. 11, 657-673.

Koerner, T.K., and Zhang, Y., (2015). "Effects of background noise on inter-trial phase coherence and auditory N1-P2 responses to speech stimuli," Hear Res. 328, 113-119.

Krishnan, A., Xu, Y., Gandour, J., and Cariani, P. (2005). "Encoding of pitch in the human brainstem is sensitive to language experience," Cogn. Brain Res. 25, 161-168.

Kuruvilla-Mathew, A., Purdy, S.C., and Welch, D. (2015) "Cortical encoding of speech acoustics: Effects of noise and amplification,” Intern. J. Audiol. 54, 852-864.

Lakatos, P., Chen, C. M., O'Connell, M. N., Mills, A., and Schroeder, C. E. (2007). "Neuronal oscillations and multisensory interaction in primary auditory cortex," Neuron 53, 279-292.

Lavitt, H. (2007). “A historical perspective on digital hearing aids: how digital technology has changed modern hearing aids." Trends Amp 11, 7-24.

Lorenzi, C., Gilbert, G., Carn, H., Garnier, S., and Moore, B. C. (2006). "Speech perception problems of the hearing impaired reflect inability to use temporal fine structure," Pro. Nat. Acad. Sci. U.S.A. 103, 18866-18869.

Lunner, T., Rudner, M., and Rönnberg, J. (2009). "Cognition and hearing aids,” Scand. J. Psychol. 50, 395-403.

Luo H., and Poeppel D. (2007). "Phase patterns of neuronal responses reliably discriminate speech in human auditory cortex," Neuron 54, 1001-1010.

Marsh, J. E., and Campbell, T. A. (2016). "Processing complex sounds passing through the 
rostral brainstem: The new early filter model," Front. Neurosci. 10, 136.

Martin, N., Lafortune, M., Godbout, J., Barakat, M., Robillard, R., Poirier, G., Bastien, C., and Carrier, J. (2013). “Topography of age-related changes in sleep spindles,” Neurobiol. Aging 34, 468-476.

Moore, B. C. (2008). "The role of temporal fine structure processing in pitch perception, masking, and speech perception for normal-hearing and hearing-impaired people," J. Assoc. Res. Otolaryngol. 9, 399-406.

Moore, B. C. (2014). Auditory processing of temporal fine structure: Effects of age and hearing loss (World Scientific).

Morillon, B., Liégeois-Chauvel, C., Arnal, L. H., Bénar, C. G., and Giraud, A. L. (2012). “Asymmetric function of theta and gamma activity in syllable processing: An intra-cortical study," Front. Psychol., 3, 248.

Ng, B. S., Logothetis, N. K., and Kayser, C. (2013). "EEG phase patterns reflect the selectivity of neural firing," Cereb. Cort. 23, 389-398.

Noguchi, Y., Fujiwara, M., and Hamano, S. (2015). “Temporal evolution of neural activity underlying auditory discrimination of frequency increase and decrease," Brain Topogr. 28, 437-444.

Oxenham, A. J., Micheyl, C., and Keebler, M. V. (2009). "Can temporal fine structure represent the fundamental frequency of unresolved harmonics?" J. Acoust. Soc. Am. 125, 2189-2199.

Peelle, J.E., Gross, J., and Davis, M.H., (2012). "Phase-locked responses to speech in human auditory cortex are enhanced during comprehension,” Cereb. Cort., 23, 1378-1387. 
Plomp, R., and Mimpen, A. M. (1979). "Speech reception threshold for sentences as a function of age and noise level,” J. Acoust. Soc. Am. 66, 1333-1342

Portas, C. M., Krakow, K., Allen, P., Josephs, O., Armony, J. L., and Frith, C. D. (2000). "Auditory processing across the sleep-wake cycle: Simultaneous EEG and fMRI monitoring in humans," Neuron 28, 991-999.

Presacco, A., Simon, J. Z., and Anderson, S. (2016). "Evidence of degraded representation of speech in noise, in the aging midbrain and cortex," J. Neurophysiol. 116, 2346-2355.

Proakis, J. G., and Manolakis, D. G. (2007). Digital Signal Processing, 4th Edition (Pearson).

Qin, M. K., and Oxenham, A. J. (2006). "Effects of introducing unprocessed low-frequency information on the reception of envelope-vocoder processed speech," J. Acoust. Soc. Am. $119,2417-26$.

Rajan, R., and Cainer, K. E. (2008). “Ageing without hearing loss or cognitive impairment causes a decrease in speech intelligibility only in informational maskers," Neuroscience $154,784-95$.

Rosen, S. (1992). "Temporal information in speech: Acoustic, auditory and linguistic aspects,” Phil. Trans. R. Soc. B: Bio. Sci. 336, 367-373.

Rosen, S., Souza, P., Ekelund, C., and Majeed, A. A. (2013) "Listening to speech in a background of other talkers: Effects of talker number and noise vocoding," J. Acoust. Soc. Am. 133, 2431-2443.

Rothauser, E. H., Chapman, W. D., Guttman, N., Nordby, K. S., Silbiger, H. R., Urbanek, G. E., and Weinstock, M. (1969). "IEEE recommended practice for speech quality measurements," IEEE Trans. Aud. Electroacoust. 17, 225-246. 
Russo, N., Nicol, T., Musacchia, G., and Kraus, N. (2004). "Brainstem responses to speech syllables," Clin. Neurophysiol. 115, 2021-2030.

Schoof, T., and Rosen, S. (2014). "The role of auditory and cognitive factors in understanding speech in noise by normal-hearing older listeners," Front. Aging Neurosci. 6:307 10.3389/fnagi.2014.00307.

Schoof, T., and Rosen, S. (2016). "The role of age-related declines in subcortical auditory processing in speech perception in noise," J. Assoc. Res. Otolaryngol. 17, 441-460.

Shannon, R. V., Zeng, F. G., Kamath, V., Wygonski, J., and Ekelid, M. (1995). "Speech recognition with primarily temporal cues," Science. 270, 303-304.

Shapiro, S. S., and Wilk, M. B. (1965). "An analysis of variance test for normality (complete samples)," Biometrika 52, 591-611.

Skoe, E., and Kraus, N. (2010). “Auditory brainstem response to complex sounds: A tutorial,” Ear Hear. 31, 302-324.

Smith, Z. M., Delgutte, B., and Oxenham, A. J. (2002). "Chimaeric sounds reveal dichotomies in auditory perception," Nature 416, 87-90.

Song, J.H., Nicol, T. and Kraus, N. (2011). "Test-retest reliability of the speech-evoked auditory brainstem response," Clin. Neurophysiol. 122, 346-355.

Song, J. H., Skoe, E., Banai, K., and Kraus, N. (2010). "Perception of speech in noise: Neural correlates,” J. Cogn. Neurosci. 23, 2268-79.

Stickney, G. S., Assmann, P .F., Chang, J., and Zeng, F. G. (2007). "Effects of cochlear implant processing and fundamental frequency on the intelligibility of competing sentences," J. Acoust. Soc. Am. 122, 1069-1078. 
Thatcher, R. W. (2012). "Coherence, phase differences, phase shift, and phase lock in EEG/ERP analyses,” Dev. Neuropsychol. 37, 476-496.

Tremblay, K. L., and Miller, C. W. (2014). "How neuroscience relates to hearing aid amplification,” Int. J. Otolaryngol. 2014, 1-7.

Turner, C. W., and Cummings, K. J. (1999). "Speech audibility for listeners with high-frequency hearing loss," Am. J. Audiol. 8, 47-56.

Turner, C. W., Gantz, B. J., Vidal, C., Behrens, A., and Henry, B. A. (2004). "Speech recognition in noise for cochlear implant listeners: Benefits of residual acoustic hearing," J. Acoust. Soc. Am. 115, 1729-1735.

Warby, S. C., Wendt, S. L., Welinder, P., Munk, E. G., Carrillo, O., Sorensen, H. B., and Mignot, E. (2014). "Sleep-spindle detection: C rowdsourcing and evaluating performance of experts, non-experts and automated methods," Nat. Meth. 11, 385-392.

Wilf, M., Ramot, M., Furman-Haran, E., Arzi, A., Levkovitz, Y., and Malach, R. (2016). "Diminished auditory responses during NREM sleep correlate with the hierarchy of language processing," PloS One 11, e0157143.

Wong, P. C., Skoe, E., Russo, N.M., Dees, T., and Kraus, N. (2007). “Musical experience shapes human brainstem encoding of linguistic pitch patterns," Nat. Neurosci. 10, 420-422.

Zeng, F. G., Nie, K., Liu, S., Stickney, G., Del Rio, E., Kong, Y. Y., and Chen, H. (2004). “On the dichotomy in auditory perception between temporal envelope and fine structure cues," J. Acoust. Soc. Am. 116, 1351-1354.

Zeng, F. G., Nie, K., Stickney, G. S., Kong, Y. Y., Vongphoe, M., Bhargave, A., Wei, C., and 
Cao, K. (2005). "Speech recognition with amplitude and frequency modulations," Pro. Nat. Acad. Sci. U.S.A. 102, 2293-2298.

Zhong, Z., Henry, K.S., and Heinz, M.G. (2014). “Sensorineural hearing loss amplifies neural coding of envelope information in the central auditory system of chinchillas," Hear. Res. $309,55-62$. 